$$
\begin{gathered}
\text { Universidade de São Paulo } \\
\text { Instituto de Física de São Carlos }
\end{gathered}
$$

Charlie Oscar Oncebay Segura

\title{
Teorias adiabáticas e aplicações em Ressonância Magnética Nuclear
}





\section{Charlie Oscar Oncebay Segura}

\section{Teorias adiabáticas e aplicações em Ressonância Magnética Nuclear}

Dissertação apresentada ao Programa de PósGraduação em Física do Instituto de Física de São Carlos da Universidade de São Paulo, para obtenção do título de mestre em Ciências.

Área de Concentração: Física Básica

Orientador: Prof. Dr. Diogo de Oliveira Soares Pinto

Versão Corrigida

(versão original disponivel na Unidade que aloja o Programa)

São Carlos 
AUTORIZO A REPRODUÇÃO E DIVULGAÇÃO TOTAL OU PARCIAL DESTE TRABALHO, POR QUALQUER MEIO CONVENCIONAL OU ELETRÔNICO, PARA FINS DE ESTUDO E PESQUISA, DESDE QUE CITADA A FONTE.

Ficha catalográfica elaborada pelo Serviço de Biblioteca e Informação do IFSC, com os dados fornecidos pelo(a) autor(a)

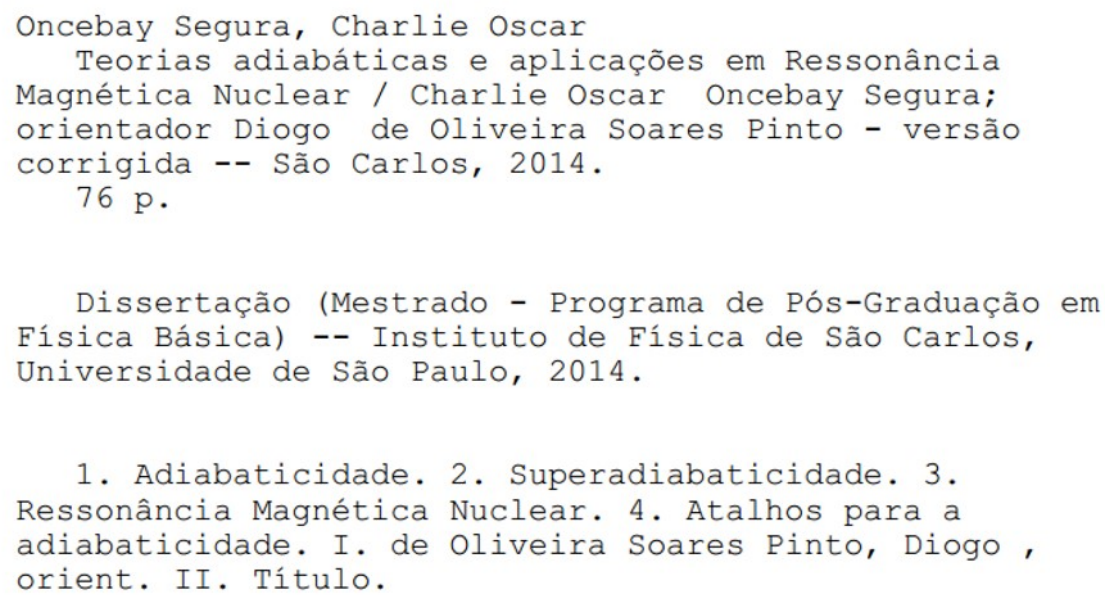

1. Adiabaticidade. 2. Superadiabaticidade. 3. Ressonância Magnética Nuclear. 4. Atalhos para a adiabaticidade. I. de Oliveira Soares Pinto, Diogo, orient. II. Título. 




\section{AGRADECIMENTOS}

Seu trabalho vai ocupar uma grande parte da sua vida, e a única maneira de estar verdadeiramente satisfeito é fazendo aquilo que você acredita ser um

ótimo trabalho. $\mathrm{E}$ a única maneira de fazer um ótimo trabalho é fazendo o que você ama fazer. Se você ainda não encontrou, continue procurando.

Steve Jobs

Gostaria de iniciar agradecendo ao meu orientador, o Prof. Dr. Diogo de Oliveira Soares Pinto, pela confiança de me aceitar como aluno, pela paciência, dedicação para ensinar e discutir os problemas. Agradeço também à sua amizade, foram dois anos de agradável convivência.

Ao Prof. Dr. Tito Bonagamba, pelo apoio, amizade, conselhos e a atenção que ele me deu em Sã Carlos.

Aos amigos que estiveram mais diretamente ligados às minhas atividades de pesquisa: Rodrigo de Oliveira Silva, Christian Rivera Ascona, Elton Tadeu Montrazi, Everton Lucas de Oliveira, Mariane Barsi Andreeta, Roberson Saraiva Polli, Arthur Gustavo de Araújo Ferreira, Daniel, Millena Logrado, Ruben Auccaise e Aparecido Amorim, obrigado a todos vocês pela companhia, ajuda, brincadeiras e piadas.

À dona Alice dona da casa onde me hospedo, eu sempre me senti como na minha casa.

Além de dedicar, eu gostaria de agradecer aos meus pais, Oscar e Zully, pela sua comprensão e apoio durante todo o mestrado e graduação. Apesar de nos encontrarmos pouco, o carinho de vocês à distância é fundamental. A educação que vocês me deram foi sem dúvida exemplar. Tudo isso permitiu, sem sombra de dúvidas, que eu pudesse chegar aqui.

Agradeço ao Instituto de Física de São Carlos pela estrutura e apoio financeiro que permitiram a realização desta pesquisa e a CAPES por me conceder a bolsa de estudos. 



\section{RESUMO}

ONCEBAY SEGURA,C.O. Teorias adiabáticas e aplicações em Ressonância Magnética Nuclear. 2014. 76 p. Dissertação (Mestrado em Ciências) - Instituto de Física de São Carlos, Universidade de São Paulo, São Carlos, 2014.

Nesta dissertação apresentaremos um estudo da adiabaticidade em Ressonância Magnética Nuclear. Para tal, apresentaremos inicialmente uma revisão sobre o conceito de adiabaticidade em sistemas quânticos fechados e abertos.

Muitos processos adiabáticos comumente utilizados em Ressonância Magnética apresentam um bom desempenho, embora a aproximação adiabática não seja valida durante todo o processo, então através do formalismo da superadiabaticidade de Berry faremos correções para que o pulso satisfaça a condição adiabática. Nesta abordagem, um Hamiltoniano dependente do tempo e que evolui lentamente é iterativamente transformado em quadros diagonais dependentes do tempo até que a aproximação adiabática mais precisa é obtida. Examinaremos as capacidades das iterações super-adiabáticas para produzir uma sequência de atalhos para a adiabaticidade do sistema e estenderemos o conceito da dinâmica superadiabática para sistemas abertos, cuja evolução é governada por uma equação mestra na forma de Lindblad, fornecendo o quadro geral necessário para determinar a estratégia de controle necessário para alcançar a superadiabaticidade.

Palavras-Chave: Adiabaticidade. Superadiabaticidade. Ressonância Magnética Nuclear. Atalhos para a adiabaticidade. 



\section{ABSTRACT}

ONCEBAY SEGURA,C.O. Adiabatic theories and applications in Nuclear Magnetic Resonance. 2014. 76 p. Dissertação (Mestrado em Ciências) - Instituto de Física de São Carlos, Universidade de São Paulo, São Carlos, 2014.

We present a study of adiabaticity in Nuclear Magnetic Resonance. First, we present a review on the concept of adiabaticity in closed and open quantum systems. Many commonly used adiabatic processes in Magnetic Resonance perform well even though the adiabatic approximation does not appear to hold throughout the process, then through the use Berry's superadiabatic formalism make corrections to the pulse satisfies the adiabatic condition. This approach, a but time-dependent Hamiltonian slowly evolving is iteratively transformed into time-dependent diagonal frames until the most accurate adiabatic approximation is obtained. Examine the capabilities of superadiabatics iterations to produce a shortcut sequence to adiabaticity system and extend the concept of dynamic superadiabatic for open systems, whose evolution is governed by a master equation of Lindblad form, providing the necessary framework to determine the strategy control needed to achieve superadibaticity.

KEYWORDS: Adiabaticity. Superadiabaticity. Nuclear Magnetic Resonance. Shortcuts to adiabaticity. 



\section{LISTA DE FIGURAS}

Figura 2.1 - Representação da indexação lógica para os niveis de spin nuclear com $I=1 / 2 \ldots \ldots \ldots \ldots \ldots \ldots \ldots$

Figura 3.1 - Campo Magnético girando em torno de $z \ldots \ldots$. . . . . . . . . . 36

Figura 3.2 - Ilustração de $C_{12}$ em função de $\omega$ e $w \ldots$. . . . . . . . . . . . . 47

Figura 4.1 - Representação gráfica dos quadros de rotação. . . . . . . . . . . . . . 54

Figura 5.1 - Gráfico de $F(t)$ comparando os dois casos, $\omega=\omega_{0}$ e $\omega=10 \omega_{0}$. . . . 59

Figura 5.2 - Gráfica de $F(t)$ para $\omega=\omega_{0}$ no segundo quadro. . . . . . . . . . . 61

Figura 5.3 - Evolução da magnetização no referencial girante. . . . . . . . . . . . . . . 64

Figura 5.4 - Evolução da magnetização no referencial girante, quando se soma ao Hamiltoniano inicial o termo $H_{C D}^{0}$. . . . . . . . . . . . . . . . . 64

Figura 5.5 - Evolução da magnetização no referencial girante, quando se soma ao Hamiltoniano inicial o termo $H_{C D}^{1}$. . . . . . . . . . . . 64

Figura 5.6 - Fator da adiabaticidade $Q_{n}$ para nosso pulso sech/tanh. . . . . . . . 65

Figura 5.7 - Gráfico da equação (5.3.12) em função de $\omega$ e $\omega_{0}^{\prime}=\omega_{1}^{\prime} . \ldots . .68$ 



\section{SUMÁRIO}

1 Introdução

2 Princípios básicos da RMN $\quad 17$

2.1 Iteração do momento magnético nuclear com campos magnéticos externos . . 18

2.2 Equação de Bloch . . . . . . . . . . . . . . . . . . . . . . . . . 22

2.3 Descrição da RMN através da matriz densidade . . . . . . . . . . . . . . . . . . 23

2.4 Abordagem da relaxação do spin nuclear com a matriz densidade . . . . . . . 25

3 Fundamentos da Adiabaticidade $\quad 29$

3.1 Introdução . . . . . . . . . . . . . . . . . . . . . . . . . . . . . 29

3.2 Fase Geométrica . . . . . . . . . . . . . . . . . . . . . . . . . . . 32

3.3 A condição para a adiabaticidade em um sistema fechado . . . . . . . . . . 34

3.4 Exemplo de fase geométrica em uma partícula de spin . . . . . . . . . . 35

3.5 Aproximação adiabática em sistemas abertos . . . . . . . . . . . . . . . 38

3.6 Blocos de Jordan de uma dimensão . . . . . . . . . . . . . . . . . . . . . . . . . 42

3.7 Exemplo: A evolução adiabática de um sistema aberto quântico de dois níveis . 43

4 Superadiabaticidade $\quad 49$

4.1 Quadros de rotação em função do tempo . . . . . . . . . . . . . . . . . . . . 49

4.2 Superadibaticidade para sistemas abertos . . . . . . . . . . . . . . . . . 54

5 Aplicações em RMN $\quad 57$

5.1 Primeira Aplicação . . . . . . . . . . . . . . . . . . . . . . 57

5.2 Segunda Aplicação . . . . . . . . . . . . . . . . . . . . . . . . 61

5.3 Terceira Aplicação . . . . . . . . . . . . . . . . . . . . . . . . . . 66 
6 Conclusões e Perspectivas

REFERÊNCIAS 


\section{CAPÍtulo 1}

\section{Introdução}

Na Mecânica Quântica, a evolução de um sistema descrito por um Hamiltoniano $H(t)$ dependente do tempo é adiabática quando as probabilidades de transição entre os autoestados dependentes do tempo de $H(t)$ são insignificantes. Isso acontece quando $H(t)$ é independente do tempo, ou quando a dinâmica é lenta em comparação com valores das escalas de tempo típicas do problema. Se o sistema evolui lentamente, o teorema adiabático ((1-4)) um dos teoremas mais antigos amplamente usados na Mecânica Quântica é satisfeito. O teorema afirma que se um estado é inicialmente preparado no n-ésimo autovetor instantâneo do $H(t)$, e o Hamiltoniano promove uma dinâmica lenta, então o estado final continuará sendo autoestado do Hamiltoniano a menos de um fator de fase. Se pode dar uma interpretação geométrica para essa dinâmica através da fase de Berry $(2,5,6)$. Em Ressonância Magnética Nuclear os processos adiabáticos desempenham um papel importante. As excitações causadas por Hamiltonianos adiabáticos permitem o monitoramento da dinâmica dos estados de spin durante o transcurso de um experimento. Este controle é necessário por exemplo para imagens por Ressonância Magnética Nuclear, a fim de garantir uma boa resolução e sensibilidade, que define a qualidade de uma imagem e, consequentemente, a fidelidade para o diagnóstico médico $(7,8)$.

No presente trabalho, estudamos não só o teorema adiabático na forma tradicional, ou seja, para sistemas fechados, mas também estudaremos o teorema adiabático para o caso de sistemas quânticos abertos, em outras palavras, sistemas quânticos acoplados com o ambiente externo. Em vez de fazer uso dos autoestados do Hamiltoniano, a adiabaticidade é definida através da forma canônica de Jordan, que leva a equação mestra, que governa a dinâmica do sistema, a uma equação semelhante à equação de Schrödinger (9-11). Esta nova perspectiva permitiu a derivação de uma aproximação adiabática que inclui o caso de sistemas em contato com ambientes externos. Esta questão é importante no contexto particular de processamento de informação quântica, onde a decoerência que causada pelo ambiente é vista como um grande obstáculo para a implementação do processamento da informação quântica $(12,13)$.

Esta dissertação encontra-se dividida em 5 capítulos, onde o primeiro corresponde à esta introdução. No capítulo 2 são expostos os princípios básicos do fenômeno de Ressonância 
Magnética Nuclear. Os tópicos tratados nesse capítulo têm como objetivo principal expor os conceitos necessários para o entendimento do fenômeno da RMN. Sendo assim, objetivando uma maior concisão do texto, assuntos importantes que não estão ligados diretamente ao assunto desta dissertação são ignorados, referenciados, ou tratados superficialmente. Esse procedimento também é adotado nos demais capítulos. O capítulo 3 e 4 entra mais especificamente no tema central do trabalho, iniciando com uma breve discussão do teorema adiabático, para depois discutir sobre a condição da adiabaticidade no sistema aberto e fechado. Também veremos como são geradas as iterações superadiabaticas e o termo chamado como contradibático. Finalmente, o quinto e último capítulo apresentamos alguns resultados das aplicações que se fizeram com Hamiltonianos típicos de Ressonância Magnética Nuclear para o estudo da adiabaticidade. 


\section{Princípios básicos da RMN}

O fenômeno da Ressonância Magnética Nuclear ( $R M N)$ pode ser observado em núcleos que possuam um momento angular total, ou spin nuclear, diferente de zero. O spin nuclear, determinado pelo operador $\hbar \mathbf{I}$, tem as mesmas propriedades de qualquer operador de momento angular da Mecânica Quântica, sendo caracterizado pelo número quântico de spin nuclear $\mathbf{I}$ $(1,2,14)$.

O momento angular total de um núcleo é devido a contribuições de todos os momentos angulares dos seus nucleons (prótons e nêutrons) constituintes. Por exemplo, se os números de prótons e de nêutrons forem ambos pares, o spin nuclear é igual a zero, indicando uma compensação entre os momentos angulares dos pares de nucleons. Se existir somente um nucleon desemparelhado, então o spin nuclear será igual ao momento angular deste único nucleon. Essa regra ajuda a entender os valores de spin nuclear de alguns núcleos tais como ${ }^{1} H(I=1 / 2),{ }^{12} C(I=1 / 2)$ e ${ }^{13} C(I=1 / 2)(15)$.

Todos os núcleos que possuem spin nuclear não nulo também possuem momento de dipolo magnético. O momento de dipolo desses núcleos está relacionado diretamente com o seu spin nuclear por $(15,16)$

$$
\mu=\gamma \hbar I
$$

sendo $\gamma$ o fator giromagnético, que é um parâmetro específico de cada espécie nuclear. As características quânticas do operador I são dadas pelo seu módulo ao quadrado e a sua componente $z$

$$
\begin{gathered}
\mathbf{I}^{2}|I, m\rangle=I(I+1)|I, m\rangle \\
\mathbf{I}_{z}|I, m\rangle=m|I, m\rangle
\end{gathered}
$$

onde $|I, m\rangle$ são os autovetores comuns a $I^{2}$ e $I_{z}$, especificados pelos números quânticos $I$ e $m$, para os quais $m=-I,-I+1, \ldots, I-1, I(14,17)$. Outros operadores essenciais para a compreensão dos fenômenos de ressonância magnética são os operadores de abaixamento e levantamento que são definidos a partir das componentes transversais como sendo respectivamente

$$
I_{-}=I_{x}-i I_{y}
$$




$$
I_{+}=I_{x}+i I_{y}
$$

e a ação desses operadores sobre os estados $|I, m\rangle$ é

$$
I_{ \pm}|I, m\rangle=\sqrt{I(I+1)+m(m \pm 1)}|I, m \pm 1\rangle .
$$

Usualmente num experimento de RMN, a magnitude da energia envolvida é muito menor do que a diferença de energia entre o estado fundamental e primeiro estado excitado dos níveis nucleares. Portanto, pode-se considerar que o núcleo permanece no estado fundamental, sendo os estados descritos por $|I, m\rangle$ com $I$ fixo. Em tais situações a energia do núcleo é determinada somente pelo número quântico $m(16,18,19)$.

Dependendo das características físicas do sistema, ele será afetado por algumas interações internas, por exemplo: o deslocamento químico que está relacionado ao ambiente eletrônico, ou seja, a forma como o movimento orbital dos elétrons próximos induz um campo magnético local ao redor do núcleo, o acoplamento dipolar devido à interação entre dois momentos de dipolo magnético de dois sistemas nucleares, o acoplamento J que é uma interação indireta entre os momentos de dipolo magnético de núcleos vizinhos, mediada pelas nuvens eletrônicas em torno das espécies nucleares e o acoplamento quadrupolar devido à interação entre o momento de quadrupolo elétrico do núcleo e o gradiente de campo elétrico existente devido à distribuição de cargas ao redor dele, esta interação ocorre apenas para núcleos com $I>1 / 2$.

Para o restante deste trabalho serão relevantes as interações externas como interação Zeeman e a interação com campos de rádio frequência que apresentaremos com mais detalhe na seguinte seção.

\subsection{Iteração do momento magnético nuclear com cam- pos magnéticos externos}

A interação básica necessária para entender o fenômeno de RMN é a chamada interação Zeeman, que ocorre entre um momento de dipolo magnético e os campos magnéticos existentes na posição do núcleo. A interação Zeeman entre dipolos magnéticos e o campo magnético estático externo quebra a degenerescência dos níveis de spin nuclear, fazendo surgir um conjunto de níveis cujas energias dependem do numero quântico m. A absorção e irradiação de energia associada às transições entre esses níveis constituem os fenômenos físicos observa- 


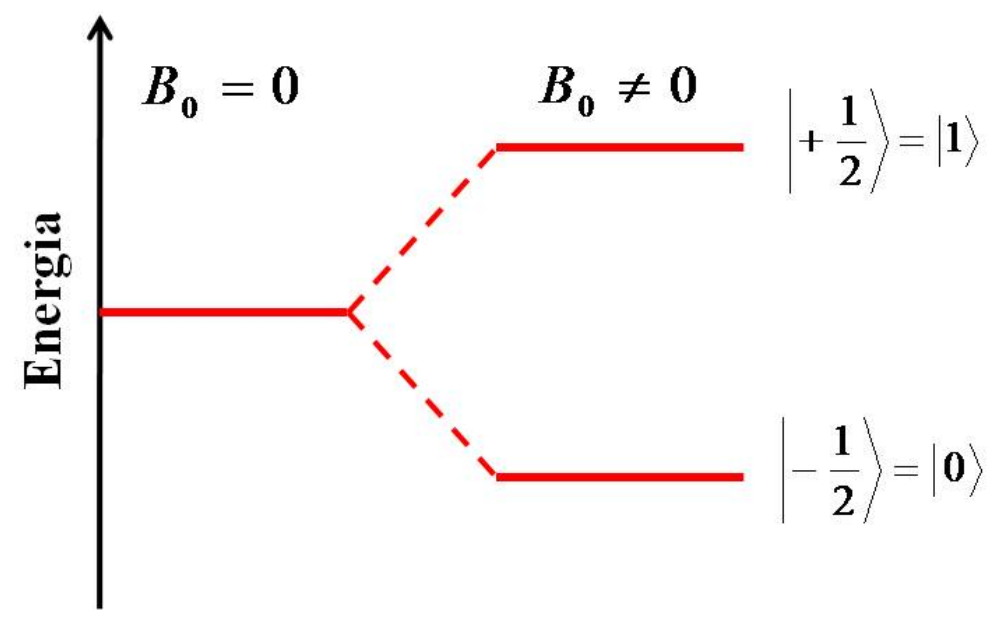

Figura 2.1-Representação da indexação lógica para os niveis de spin nuclear com $I=1 / 2$.

Fonte: Elaborada pelo autor.

dos nos experimentos de RMN. Assim, quando um núcleo atômico com momento de dipolo magnético dado por (2.0.1) é colocado em um campo magnético estático $B_{0}$, os diferentes estados de spin nuclear $|m\rangle$ passam a possuir energias diferentes, sendo esse efeito denominado efeito Zeeman nuclear $(16,20)$. Quanticamente o efeito Zeeman nuclear é descrito pelo seguinte Hamiltoniano

$$
H_{z}=-\mu_{z} B_{0}=-\gamma \hbar B_{0} I_{z}=-\hbar \omega_{L} I_{z}
$$

sendo $\omega_{L}$ a denominada frequência de Larmor. A direção $z$ corresponde ao eixo definido pelo campo magnético $B_{0}$ e todos os operadores quânticos agem no subespaço expandido por $|m\rangle$ onde $m=-I,-I+1, \ldots, I-1, I$. Os autovalores do Hamiltoniano Zeeman (2.1.1), são proporcionais aos autovalores do operador $I_{z}$,

$$
E_{m}=-m \hbar \omega_{L}
$$

Portanto, a interação Zeeman é responsável pela quebra da degenerescência dos níveis de energia nucleares de forma que para um spin nuclear $I$ existem $2 I+1$ níveis de energia. Para um spin com $I=1 / 2$, existem dois estados com níveis de energia diferentes. Podemos fazer uma indexação lógica e escrever o estado de energia mais baixa como $|0\rangle$ e o estado de energia mais alta como $|1\rangle$. Assim estamos atribuindo a dois níveis quânticos de energia distinta dois valores lógicos distintos, esse spin pode ser usado então para representar um bit quântico, o q-bit. A transição entre os níveis de energia pode ser induzida através da aplicação de campos magnéticos oscilantes, cuja intensidade é tipicamente muito menor do que $B_{0}$, com apropriada frequência de Larmor. Para os spins nucleares, a frequência de Larmor varia de alguns $\mathrm{MHz}$ até alguns $\mathrm{GHz}$ e, portanto, a excitação é obtida através de um campo de radio frequência 
(RF) na mesma faixa (18). Essa excitação dos estados quânticos dos spins nucleares pode ser entendida considerando o efeito de um segundo campo magnético dependente do tempo, $B_{1}(t)$, aplicado perpendicularmente ao campo magnético estático $B_{0}$, do tipo

$$
B_{1}(t)=B_{1}(\hat{i} \cos \omega t+\hat{j} \operatorname{sen} \omega t)
$$

onde $\omega$ é a frequência do campo de RF e $\hat{i}$ e $\hat{j}$ são os vetores unitários ao longo das direções $x$ e $y$. Assim o Hamiltoniano de RF é dado por

$$
H_{R F}=-\mu \cdot B_{1}(t)=-\hbar \omega_{1}\left(I_{x} \cos \omega t+I_{y} \operatorname{sen} \omega t\right)
$$

onde $\omega_{1}=\gamma B_{1}$ e $I_{x}$ e $I_{y}$ são, respectivamente, as componentes $x$ e $y$ do operador de spin nuclear $I$. Logo, o Hamiltoniano total do sistema, Zeeman mais a interação com a radio frequência, fica

$$
H=-\hbar \omega_{L} I_{z}-\hbar \omega_{1}\left(I_{x} \cos \omega t+I_{y} \operatorname{sen} \omega t\right)
$$

Agora vejamos como a equação (2.1.5) se transforma sob a mudança para um referencial de coordenadas girantes (RG) cuja componente z coincide com o referencial do laboratório (RL). Seja $\omega$ a frequência de rotação do $\mathrm{RG}$ em relação ao $\mathrm{RL}$, então $|\psi(t)\rangle$ representado no $\mathrm{RG}$ é $|\varphi(t)\rangle=e^{i \omega t I_{z}}|\psi(t)\rangle$. Com isso a equação de Schrödinger do sistema

$$
i \hbar \frac{\partial|\psi(t)\rangle}{\partial t}=H(t)|\psi(t)\rangle
$$

poder ser reescrita como

$$
i \frac{\partial|\varphi(t)\rangle}{\partial t}=\left[e^{i \omega t I_{z}} H e^{-i \omega t I_{z}}-\omega I_{z}\right]|\varphi(t)\rangle
$$

e usando a seguinte relação $(21,22)$

$$
\begin{aligned}
& e^{-i \theta I_{x}} I_{y} e^{i \theta I_{x}}=I_{y} \cos \theta+I_{z} \operatorname{sen} \theta \\
& e^{-i \theta I_{y}} I_{z} e^{i \theta I_{y}}=I_{z} \cos \theta+I_{x} \operatorname{sen} \theta \\
& e^{-i \theta I_{z}} I_{x} e^{i \theta I_{z}}=I_{x} \cos \theta+I_{y} \operatorname{sen} \theta
\end{aligned}
$$

obtemos

$$
i \frac{\partial|\varphi(t)\rangle}{\partial t}=\left[\left(\omega_{L}+\omega\right) I_{z}+\omega_{1} I_{x}\right]|\varphi(t)\rangle
$$

Então o Hamiltoniano efetivo experimentado pelo spin no RG é descrito por

$$
H_{R G}=-\hbar\left[\left(\omega_{L}+\omega\right) I_{z}+\omega_{1} I_{x}\right]
$$


Assim, se $\omega=-\omega_{L}$ então o campo $B_{1}$ está em ressonância com o sistema e, por tanto, apesar de possuir intensidade muito menor que o campo estático $\left(B_{1}<<B_{0}\right)$, apenas o seu efeito é sentido pelo spin (16).

No referencial RG, $H_{R G}$ é independente do tempo, então a solução da equação de Schrödinger é

$$
|\varphi(t)\rangle=e^{-i H_{R G} t / \hbar}|\varphi(0)\rangle
$$

Já no RL a solução é

$$
|\psi(t)\rangle=e^{-i \omega t I_{z}} e^{-i H_{R G} t / \hbar}|\psi(0)\rangle .
$$

Supondo que o campo $B_{1}$ foi aplicado em ressonância com o sistema, então $H_{R G}=-\hbar \omega I_{x}$ e a magnetização média na direção $z$ será dada por

$$
\begin{gathered}
\left\langle\mu_{z}(t)\right\rangle=\int\left\langle\psi(t)\left|\mu_{z}\right| \psi(t)\right\rangle d r^{3} \\
\left\langle\mu_{z}(t)\right\rangle=\hbar \gamma \int\left\langle\psi(0)\left|e^{-i \omega_{1} t I_{x}} e^{i \omega t I_{z}} I_{z} e^{-i \omega t I_{z}} e^{i \omega_{1} t I_{x}}\right| \psi(0)\right\rangle d r^{3}
\end{gathered}
$$

. Usando a propriedade (2.1.8), obtemos:

$$
\left\langle\mu_{z}(t)\right\rangle=\left\langle\mu_{z}(0)\right\rangle \cos \omega_{1} t-\left\langle\mu_{y}(0)\right\rangle \operatorname{sen} \omega_{1} t
$$

Se em $t=0$ a magnetização está ao longo do eixo $z$, então $\langle\mu(0)\rangle=0$, portanto

$$
\left\langle\mu_{z}(t)\right\rangle=\left\langle\mu_{z}(0)\right\rangle \cos \omega_{1} t
$$

Então no RG, observamos o momento magnético precessionando em torno do eixo $x$ com freqwência angular $\omega_{1}$. No caso de um sistemas com spins que possuam frequências características, $\omega_{L}$, diferentes, cada spin possui o seu próprio referencial girante, com sua respectiva freqüência de rotação (23). 


\subsection{Equação de Bloch}

Os momentos magnéticos nucleares contidos em um agregado de matéria estão sujeitos à agitação térmica e à flutuação do campo magnético local devido aos momentos vizinhos, fazendo com que a magnetização total de um agregado de matéria sofra alterações em sua dinâmica, além daquela determinada pelo campo externo $B_{0}$. Em 1946, Félix Bloch (24) propôs um modelo fenomenológico para a dinâmica da magnetização. Esse modelos baseia-se num conjunto de equações conhecido como Equações de Bloch, que expressas no referencial girante de coordenadas são dadas por:

$$
\begin{aligned}
\frac{d M_{x}}{d t} & =\gamma M_{y}\left(B_{0}-\frac{\omega}{\gamma}\right)-\frac{M_{x}}{T_{2}} \\
\frac{d M_{y}}{d t} & =\gamma M_{z} B_{1}-\gamma M_{x}\left(B_{0}-\frac{\omega}{\gamma}\right)-\frac{M_{y}}{T_{2}} \\
\frac{d M_{z}}{d t} & =-\gamma M_{y} B_{1}-\frac{M_{z}-M_{0}}{T_{1}}
\end{aligned}
$$

onde $M_{0}$ é o módulo da magnetização de equilibrio e $T_{1}$ e $T_{2}$ são chamados de tempo de relaxação longitudinal e transversal, respectivamente. A relaxação transversal da magnetização ocorre devido à criação de um campo pelo própio spin nuclear no sítio de outros núcleos, o que gera uma heterogeneidade instantânea no campo magnético acarretando na perda de coerência de fase macroscópica. A relaxação longitudinal da magnetização está relacionada com as transições entre os níveis de energia do spin nuclear, a tendência do sistema é liberar o excesso de energia com o ambiente magnético ao redor.

Vamos resolver a equação (2.2.1) para o caso de um campo estático $\vec{B}=B_{0} \hat{k}$, com a condição inicial $\vec{M}(0)=M_{0} \hat{i}^{\prime}$ no referencial girante com a frequência de Larmor. Essa condição inicial pode ser obtida aplicando-se um pulso de exitação sobre uma magnetização de equilibrio $\vec{M}=M_{0} \hat{k}$. Encontramos as soluções no referencial do laboratório como sendo $(16,21)$

$$
\begin{aligned}
& M_{x}(t)=M_{0} \cos \left(\omega_{0} t\right) e^{-t / T_{2}} \\
& M_{y}(t)=M_{0} \operatorname{sen}\left(\omega_{0} t\right) e^{-t / T_{2}} \\
& M_{z}(t)=M_{0}\left[1-e^{-t / T_{1}}\right] \cos \left(\omega_{0} t\right) e^{-t / T_{1}}
\end{aligned}
$$

Podemos escrever as componentes $x$ e $y$ utilizando notação complexa, definido assim a magnetização transversal $\left(M_{T}\right)$ 


$$
M_{T}(t)=M_{x}(t)+i M_{y}(t)=M_{0} e^{i \omega_{0} t} e^{-t / T_{2}}
$$

\subsection{Descrição da RMN através da matriz densidade}

Uma partícula pode ser colocada em um estado quântico bem determinado utilizando algum aparato experimental. Esse estado é um estado puro. Se consideramos um sistema com várias partículas, tendo todas elas sido colocadas em um estado idêntico, o sistema continua sendo puro e pode ser representado pelo mesmo vetor de estado. Já se cada partícula for colocada em um estado diferente das outras, o sistema dado pela soma das partículas não pode ser mais considerado puro, e também não pode ser representado por um único vetor de estado. Nesse caso temos uma mistura estatística, chamada também de estado misto.

Sistemas de RMN não constituem estados puros, ao contrário são misturas estatísticas, onde a magnetização observada corresponde, a uma média sobre o ensemble de momentos magnéticos dos núcleos da amostra. Por esta razão, o formalismo de Operador Densidade torna-se uma ferramenta essencial na descrição deste tipo de sistema (14).

Se temos um estado representado no espaço de Hilbert da seguinte forma $|\psi\rangle=\sum c_{i}\left|\varphi_{i}\right\rangle$, podemos chegar à seguinte relação para o valor médio do observável $A$

$$
\begin{aligned}
\langle A\rangle & =\langle\psi|A| \psi\rangle \\
& =\sum_{i j}\left\langle\varphi_{j}|A| \varphi_{i}\right\rangle c_{i} c_{j}^{*} \\
& =\sum_{i j}\left\langle\varphi_{j}|A| \varphi_{i}\right\rangle\left\langle\varphi_{i}|| \psi\right\rangle\left\langle\psi|| \varphi_{j}\right\rangle \\
& =\sum_{j}\left\langle\varphi_{j}|A \rho| \varphi_{j}\right\rangle \\
\langle A\rangle & =\operatorname{Tr}\{A \rho\}
\end{aligned}
$$

onde temos que o estado pode ser completamente representado por uma matriz $\rho$

$$
\rho=|\psi\rangle\langle\psi|
$$


com os seguintes termos

$$
\rho_{i j}=\left\langle\varphi_{j}|\rho| \varphi_{i}\right\rangle=c_{i} c_{j}^{*}
$$

Essa matriz é chamada de matriz densidade. Seus termos diagonais, em que $i=j$, são chamados populações e representam a probabilidade de medida de cada auto-estado, $\left|c_{i}^{2}\right|$. Os termos fora da diagonal, $i \neq j$, são chamados coerências e são os termos de interferência entre os diferentes autoestado.

Os operadores densidade são sempre hermitianos e de traço igual a um. A evolução temporal do operador densidade do estado $\psi$ pode ser obtida mediante a equação de Liouvillevon Neumann (14)

$$
i \hbar \frac{\partial \rho}{\partial t}=[H, \rho]
$$

Se cada partícula for colocada em um estado diferente das outras, o sistema dado pela soma das partículas não pode ser mais considerado puro e também não pode mais ser representado por um único vetor de estado. Nesse caso temos uma mistura estatística, chamada também de estado misto, e o operador densidade fica dado por

$$
\rho=\sum_{i=1}^{N} p_{i}\left|\psi_{i}\right\rangle\left\langle\psi_{i}\right|
$$

No equilíbrio térmico, o operador densidade da mistura estatística pode ser escrito em termos do Hamiltoniano e sua função de partição $Z=\operatorname{Tr}\left\{\exp \left(-H / k_{B} T\right)\right\}$, então

$$
\rho_{0}=\frac{e^{-H / k_{B} T}}{Z}
$$

neste caso, as populações satisfazem a distribuição de Boltzmann (25).

No caso de um ensemble de núcleos idênticos, com número quântico $I$, e na presença de um campo $B_{0}$ aplicado na direção $z$, o Hamiltoniano Zeeman é $H_{z}=-\hbar \omega_{L} I_{z}$. Para os campos magnéticos e temperaturas típicas dos experimentos atuais de RMN encontramos que $\frac{\hbar \omega_{L}}{k_{B} T} \approx 10^{-5}$, o que torna a aproximação de altas temperaturas $\left(k_{B} T>>\hbar \omega_{L}\right)$ viável, então a matriz densidade de equilibrio pode ser escrita como

$$
\rho_{0}=\left(\frac{1}{2 I+1}\right)+\left(\frac{\alpha}{2 I+1}\right) I_{z}
$$

sendo $\alpha=\hbar \omega_{L} / k_{B} T$. O primeiro termo da equação (2.3.11) não contribui para o sinal de RMN e nem é sensível a ação dos pulsos de RF, por isso o segundo termo é denominado como 
Matriz Densidade de Desvio $(\Delta \rho)$, e é a única parte que efetivamente contribui nas análises por RMN. Podemos dizer que esta da matriz densidade contém toda a informação relevante do sistema (26-28).

\subsection{Abordagem da relaxação do spin nuclear com a ma- triz densidade}

Esse processo, que pode ser chamado de processo de relaxação também pode ser descrito usando o formalismo do operador densidade $(22,29)$. Um obstáculo para o controle do sistema é a incapacidade de se isolar completamente o sistema dos efeitos do ambiente em que se encontra. Inevitavelmente, após de um certo tempo, a interação do sistema com o ambiente induzirá misturas no estado inicial $(22,29)$. Por tanto, modelos que descrevam tal dinâmica quântica devem levar em consideração os efeitos do ambiente.

Seja o sistema quântico descrito pelo operador densidade $\rho_{S}$ e um ambiente descrito pelo operador densidade $\rho_{A}$. Seja também o estado conjunto inicial dado por $\rho_{S A}=\rho_{S} \otimes \rho_{A}$. A evolução do sistema em conjunto é dada pelo operador unitário $U(t)$ de forma que

$$
\rho_{S A}=U(t) \rho_{S} \rho_{A} U^{\dagger}(t)
$$

Para obter a descrição somente da evolução do sistema deve-se realizar a operação quântica

$$
\rho_{S}(t)=\epsilon\left(\rho_{S A}\right)=\operatorname{Tr}_{A}\left[U(t) \rho_{S} \rho_{A} U^{\dagger}(t)\right]
$$

onde $\operatorname{Tr}_{A}$ é o traço parcial sobre o espaço de Hilbert do ambiente, e $\rho_{S}(t)$ é o operador densidade reduzido. Sem perda de generalidade, supondo que o estado inicial do ambiente é $\rho_{A}=\left|e_{0}\right\rangle\left\langle e_{0}\right|$, tem-se (22)

$$
\begin{gathered}
\epsilon\left(\rho_{S A}\right)=\rho_{S}=\sum_{k}\left\langle e_{k}\left|U\left(\rho_{S} \otimes\left|e_{0}\right\rangle\left\langle e_{0}\right|\right) U^{\dagger}\right| e_{k}\right\rangle \\
\left.\epsilon\left(\rho_{S A}\right)=\rho_{S}=\sum_{k}\left\langle e_{k}|U| e_{0}\right\rangle \rho_{S}\left\langle e_{0}\right|\right) U^{\dagger}\left|e_{k}\right\rangle \\
\epsilon\left(\rho_{S A}\right)=\rho_{S}=\sum_{k} A_{k} \rho_{S} A_{k}^{\dagger}
\end{gathered}
$$


onde $A_{k}=\left\langle e_{k}|U| e_{0}\right\rangle$ é conhecido como operador de Kraus e a equação (2.4.3) é conhecida como a representação de operador soma (22), com a condição de normalização:

$$
\sum_{k} A_{k}^{\dagger} A_{k}=\sum_{k}\left\langle e_{0}\left|U^{\dagger}\right| e_{k}\right\rangle\left\langle e_{k}|U| e_{0}\right\rangle=\mathbb{I}
$$

É importante notar que os operadores de Kraus não são definidos de forma única. Olhando para a equação (2.4.3) vê-se que, caso mude a base sob a qual se opera o traço, os operadores de Kraus irão mudar, também os operadores de Kraus descrevem a dinâmica do sistema principal sem considerar explicitamente as propriedades do ambiente. Portanto, a partir dessa descripção da evolução do operador densidade é possível descrever a relaxação dos spins nucleares através da atenuação de amplitude e da atenuação de fase (22). Vale ressaltar que o processo de relaxação de diferentes sistemas pode ser descrita pelo mesmo conjunto de operadores, visto que o foco deste formalismo está somente na descripção do sistema principal e não na maneira como ocorre a interação com o banho térmico (22).

Nos sistemas de RMN, a atenuação da amplitude está ligada à relaxação longitudinal à temperaturas finitas. Os operadores de Kraus que descreven esse efeito são dados por

$$
\begin{gathered}
A_{1}=\sqrt{p}\left[\begin{array}{rr}
1 & 0 \\
0 & \sqrt{1-\gamma}
\end{array}\right], A_{2}=\sqrt{p}\left[\begin{array}{rr}
0 & \sqrt{\gamma} \\
0 & 0
\end{array}\right] \\
A_{3}=\sqrt{1-p}\left[\begin{array}{rr}
\sqrt{1-\gamma} & 0 \\
0 & 1
\end{array}\right], A_{4}=\sqrt{1-p}\left[\begin{array}{rr}
0 & 0 \\
\sqrt{\gamma} & 0
\end{array}\right]
\end{gathered}
$$

onde $p \approx \frac{1}{2}-\frac{\hbar \omega_{L}}{2 k_{B} T}$ é o peso de Boltzmann, nesse caso dado por esses operadores que podem ser relacionados a um processo na qual um q-bit em um estado excitado decai para o estado fundamental com probabilidade $\gamma p$ e sai do estado fundamental para o excitado com probabilidade $[1-\gamma p](28)$.

Em contrapartida,a atenuação de fase está ligada à relaxação transversal. Para este caso, os operadores de Kraus que descrevem tal efeito são dados por:

$$
A_{1}=\sqrt{\lambda}\left[\begin{array}{ll}
1 & 0 \\
0 & 1
\end{array}\right], A_{2}=\sqrt{1-\lambda}\left[\begin{array}{rr}
1 & 0 \\
0 & -1
\end{array}\right]
$$

No caso de um sistema de 2 spins $1 / 2$ não interagentes, estes canais são descritos pelos operadores de Kraus aprensentados anteriormente, onde $\alpha$, e $\gamma$ são obtidos a partir das equações de Bloch (22). Assim, para canal de atenuação de amplitude generalizada e para a 
atenuação de fase se relaciona à relaxação transversal do sistema respectivamente tem-se:

$$
\begin{gathered}
\gamma=1-e^{-t / T_{1}} \\
\lambda=\frac{1}{2}\left(1-e^{-t / 2 T_{2}}\right)
\end{gathered}
$$

sendo $T_{1}, T_{2}$ os tempos de relaxação longitudianal e transversal respectivamente, característico deste processo. 


\section{Fundamentos da Adiabaticidade}

A adiabaticidade é uma das questões mais antigas em Mecânica Quântica e foi fundamental no desenvolvimento de novas ferramentas como as fases de Berry (30), que dependem somente da geometria do caminho percorrido durante a evolução. Há poucos anos o teorema adiabático voltou a ganhar atenção na área de informação quântica devido ao papel que tem na computação adiabática (12), (13). A primeira formulação e demonstração do teorema foram feitas por Born e Fock em 1928 (3) e foram motivadas pelo trabalho de Ehnrenfest de 1916 (4), onde se relacionam invariantes adiabáticos clássicos com os correspondentes observáveis quânticos. Neste capítulo mostraremos o teorema adiabático e veremos a sua importância quando o utilizarmos nas aproximações adiabáticas para o caso do sistema fechado e aberto.

\subsection{Introdução}

O teorema adiabático (2) afirma que se em $t=0$ um sistema quântico é preparado no n-ésimo autovetor do Hamiltoniano $H(t=0)$ e o mesmo varia de maneira suficientemente lenta, o sistema vai ficar em um estado próximo ao n-ésimo autovetor instantâneo deste Hamiltoniano com o passar do tempo a menos de um fator de fase multiplicativo. Portanto a mudança no Hamiltoniano não precisa ser pequena, tudo o que tem que satisfazer é que aconteça lentamente.

Vamos considerar que os níveis de energia são discretos e não degenerados em toda a transição do Hamiltoniano inicial até o Hamiltoniano final e a evolução do Hamiltoniano é governado pela equação de Schrödinger

$$
i \hbar \frac{d|\psi(t)\rangle}{d t}=H(t)|\psi(t)\rangle
$$

onde o Hamiltoniano dependente do tempo é dado por

$$
H(t)\left|\varphi_{n}(t)\right\rangle=E_{n}(t)\left|\varphi_{n}(t)\right\rangle
$$


sendo $\left|\varphi_{m}(t)\right\rangle$ a base de autoestados instantânea que satisfaz a relação

$$
\left\langle\varphi_{n}(t) \mid \varphi_{m}(t)\right\rangle=\delta_{n m}
$$

Para resolver a equação de Schrödinger consideramos o seguinte ansatz

$$
|\psi(t)\rangle=\sum_{n} c_{n}(t)\left|\varphi_{n}(t)\right\rangle e^{i \theta_{n}(t)}
$$

sendo

$$
\theta_{n}(t)=-\frac{1}{\hbar} \int_{0}^{t} E_{n}\left(t^{\prime}\right) d t^{\prime}
$$

Adiante ficará clara a motivação deste ansatz e sua interpretação física. Substituindo o ansatz na equação (3.1.1) obtemos

$$
\begin{aligned}
i \hbar \sum_{n}\left(\dot{c}_{n}\left|\varphi_{n}\right\rangle+c_{n}\left|\dot{\varphi}_{n}\right\rangle+i c_{n}\left|\varphi_{n}\right\rangle \dot{\theta}\right) e^{i \theta_{n}(t)} & =\sum_{n} c_{n} H\left|\varphi_{n}\right\rangle e^{i \theta_{n}(t)} \\
i \hbar \sum_{n}\left(\dot{c}_{n}\left|\varphi_{n}\right\rangle+c_{n}\left|\dot{\varphi}_{n}\right\rangle+i c_{n}\left|\varphi_{n}\right\rangle \dot{\theta}\right) e^{i \theta_{n}(t)} & =\sum_{n} c_{n} E_{n}\left|\varphi_{n}\right\rangle e^{i \theta_{n}(t)} \\
i \hbar \sum_{n}\left(\dot{c}_{n}\left|\varphi_{n}\right\rangle+c_{n}\left|\dot{\varphi}_{n}\right\rangle+i c_{n}\left|\varphi_{n}\right\rangle \dot{\theta}\right) e^{i \theta_{n}(t)} & =-\hbar \sum_{n} c_{n}\left|\varphi_{n}\right\rangle \dot{\theta}_{n} e^{i \theta_{n}(t)} \\
\sum_{n}\left(\dot{c}_{n}\left|\varphi_{n}\right\rangle+c_{n}\left|\dot{\varphi}_{n}\right\rangle\right) e^{i \theta_{n}(t)} & =0
\end{aligned}
$$

Se agora projertamos sobre $\left|\varphi_{m}\right\rangle$, encontramos

$$
\begin{gathered}
\sum_{n} \dot{c}_{n} \delta_{n m} e^{i \theta_{n}(t)}=-\sum_{n} c_{n}\left\langle\varphi_{m} \mid \dot{\varphi}_{n}\right\rangle e^{i \theta_{n}(t)} \\
\dot{c}_{n}=-\sum_{n} c_{n}\left\langle\varphi_{m} \mid \dot{\varphi}_{n}\right\rangle e^{i\left(\theta_{n}(t)-\theta_{m}(t)\right)} .
\end{gathered}
$$

Derivando a equação (3.1.2)

$$
\dot{H}\left|\varphi_{n}\right\rangle+H\left|\dot{\varphi}_{n}\right\rangle=\dot{E}_{n}\left|\varphi_{n}\right\rangle+E_{n}\left|\dot{\varphi}_{n}\right\rangle
$$

e projetando sobre $\left|\varphi_{m}\right\rangle$ encontraremos

$$
\begin{aligned}
\left\langle\varphi_{m}|\dot{H}| \varphi_{n}\right\rangle+\left\langle\varphi_{m}|H| \dot{\varphi}_{n}\right\rangle & =\dot{E}_{n} \delta_{m n}+E_{n}\left\langle\varphi_{m} \mid \dot{\varphi}_{n}\right\rangle \\
\left\langle\varphi_{m}|\dot{H}| \varphi_{n}\right\rangle+E_{m}\left\langle\varphi_{m} \mid \dot{\varphi}_{n}\right\rangle & =\dot{E}_{n} \delta_{m n}+E_{n}\left\langle\varphi_{m} \mid \dot{\varphi}_{n}\right\rangle
\end{aligned}
$$

Para $m=n$

$$
\left\langle\varphi_{m}|\dot{H}| \varphi_{n}\right\rangle=0
$$

e para $m \neq n$

$$
\left\langle\varphi_{m}|\dot{H}| \varphi_{n}\right\rangle=\left(E_{n}-E_{m}\right)\left\langle\varphi_{m} \mid \dot{\varphi}_{n}\right\rangle
$$


Substituindo na equação (3.1.5), encontramos

$$
\dot{c}_{m}=-c_{m}\left\langle\varphi_{m} \mid \dot{\varphi}_{m}\right\rangle-\sum_{n \neq m} c_{n} \frac{\left\langle\varphi_{m}|\dot{H}| \varphi_{n}\right\rangle}{E_{n}-E_{m}} e^{i\left(\theta_{n}(t)-\theta_{m}(t)\right)}
$$

cuja integração nos dá

$$
c_{m}(t)=c_{m}(0)-\int_{0}^{t} c_{m}(s)\left\langle\varphi_{m} \mid \dot{\varphi}_{m}\right\rangle d s-\sum_{n \neq m} \int_{0}^{t} c_{n}(s) \frac{\left\langle\varphi_{m}|\dot{H}| \varphi_{n}\right\rangle}{E_{n}-E_{m}} e^{i\left(\theta_{n}(s)-\theta_{m}(s)\right)} d s
$$

Focando nossa atenção na integral que aparece no último termo do lado direito da equação anterior

$$
I=\int_{0}^{t} c_{n}(s) \frac{\left\langle\varphi_{m}|\dot{H}| \varphi_{n}\right\rangle}{E_{n}-E_{m}} e^{i\left(\theta_{n}(s)-\theta_{m}(s)\right)} d s .
$$

Podemos a definir a variável adimensional $\tau$ como

$$
\tau=\frac{t}{T}
$$

sendo $T$ o tempo de duração do pulso. Então

$$
I=\int_{0}^{\tau} c_{n}\left(s_{1}\right) \frac{\left\langle\varphi_{m}|\dot{H}| \varphi_{n}\right\rangle}{\left[E_{n}\left(s_{1}\right)-E_{m}\left(s_{1}\right)\right]} \exp \left[-\frac{i T}{\hbar} \int_{0}^{s_{1}}\left[E_{n}\left(s_{1}\right)-E_{m}\left(s_{1}\right) d s^{\prime}\right]\right],
$$

sendo

$$
g(\tau)=\frac{1}{\hbar} \int_{0}^{\tau} d s_{1}\left[E_{m}\left(s_{1}\right)-E_{n}\left(s_{1}\right)\right] .
$$

Então, a integral (3.1.11) pode ser reescrita como

$$
I=-\int_{g(0)}^{g(\tau)} c_{n}(g) \frac{\left\langle\varphi_{m}|\dot{H}| \varphi_{n}\right\rangle}{\dot{g}} e^{i T g} d g .
$$

Para poder resolver a equação da acima, vamos lembrar o teorema de Riemann-Lebesgue (31) o qual nos afirma que

$$
\lim _{k \rightarrow \infty} \int_{a}^{b} F(x) e^{i k x} d x=0
$$

onde $F(x)$ é uma função contínua no intervalo $[a, b]$.

Observando que a integral de (3.1.13) deve ser realizada no limite adiabático, $T \rightarrow \infty$, podemos reescrevê-la, para $n \neq m$, como

$$
I=-\lim _{T \rightarrow \infty} \frac{1}{\hbar} \int_{g(0)}^{g(\tau)} c_{n}(g) \frac{\left\langle\varphi_{m}|\dot{H}| \varphi_{n}\right\rangle}{\dot{g}} e^{i T g} d g
$$

onde estamos usando a notação $\dot{g}=d g(\tau) / d \tau$. Logo, usando o teorema de Riemann-Lebesgue, obtêm-se que $I=0$. Assim, no limite em que $T \rightarrow \infty$, temos

$$
\dot{c}_{m}=-c_{m}\left\langle\varphi_{m} \mid \dot{\varphi}_{m}\right\rangle,
$$


cuja solução da equação diferencial fica dada por

$$
c_{m}(t)=c_{m}(0) \exp \left(-\int_{0}^{t}\left\langle\varphi_{m} \mid \dot{\varphi}_{m}\right\rangle d t^{\prime}\right) .
$$

Da ortonormalidade $\left\langle\varphi_{n}(t) \mid \varphi_{n}(t)\right\rangle=1$, temos que

$$
\left\langle\dot{\varphi}_{n}(t) \mid \varphi_{n}(t)\right\rangle+\left\langle\varphi_{n}(t) \mid \dot{\varphi}_{n}(t)\right\rangle=0
$$

e a partir da relação: $\left\langle\dot{\varphi}_{n} \mid \varphi_{m}\right\rangle=\left\langle\varphi_{m} \mid \dot{\varphi}_{n}\right\rangle^{*}$, temos que $\left\langle\dot{\varphi}_{n} \mid \varphi_{m}\right\rangle$ é um termo puramente imaginário. Por isso definimos

$$
\gamma_{m}(t)=i \int_{0}^{t}\left\langle\varphi_{m}(t) \mid \dot{\varphi}_{m}(t)\right\rangle d t^{\prime}
$$

Então

$$
c_{m}(t)=c_{m}(0) e^{i \gamma_{m}(t)}
$$

que substituído na equação (3.1.3), nos dá

$$
|\psi(t)\rangle=\sum_{n} c_{n}(0) e^{i \gamma_{n}(t)} e^{i \theta_{n}(t)}\left|\varphi_{n}(t)\right\rangle
$$

Como caso particular vamos considerar que $c_{n}(0)=1$ e $c_{m}(0)=0$ para $m \neq n$, logo

$$
|\psi(t)\rangle=e^{i \gamma_{n}(t)} e^{i \theta_{n}(t)}\left|\varphi_{n}(t)\right\rangle
$$

Podemos notar que o sistema se mantém no n-ésimo estado, adquirindo apenas um fator de fase.

\subsection{Fase Geométrica}

Berry observou que (30), para o caso do transporte adiabático ao longo de uma trajetória fechada, o fator de fase da função de estado não depende simplesmente da evolução temporal do Hamiltoniano. A função de onda além do fator de fase dinâmica, que depende da evolução, retém um fator de fase conhecido como fase geométrica que depende da trajetória no espaço de Hilbert projetivo, espaço dos estados a menos das fases, no caso particular da fase de Berry é o espaço dos parâmetros que fazem mudar os estados $\left|\varphi_{n}\right\rangle$. Assim, a fase total adquirida por um estado após uma evolução é a soma das fases geométrica e dinâmica. Portanto se o 
Hamiltoniano retorna ao estado inicial depois de un tempo $T$, da equação (3.1.19), temos

$$
|\psi(T)\rangle=e^{i \gamma_{n}(T)} e^{i \theta_{n}(T)}\left|\varphi_{n}(T)\right\rangle
$$

onde $\theta_{n}(T)$ é a fase dinâmica e $\gamma_{n}(T)$ é a fase geométrica

$$
\begin{gathered}
\theta_{n}(T)=-\frac{1}{\hbar} \int_{0}^{T} E_{n}\left(t^{\prime}\right) d t^{\prime} \\
\gamma_{n}(T)=i \int_{0}^{T}\left\langle\varphi_{n}\left(t^{\prime}\right) \mid \dot{\varphi}_{n}\left(t^{\prime}\right)\right\rangle d t^{\prime} .
\end{gathered}
$$

O estado $\left|\varphi_{n}\right\rangle$ depende do tempo $t$ porque existe algum parâmetro, $R(t)$, no Hamiltoniano que está variando com o tempo. Então podemos fazer

$$
\frac{\partial \varphi_{n}}{\partial t}=\frac{\partial \varphi_{n}}{\partial R} \frac{d R}{d t}
$$

Assim

$$
\gamma_{n}(t)=i \int_{0}^{t}\left\langle\varphi_{n}\left|\frac{\partial}{\partial R}\right| \varphi_{n}\right\rangle \frac{d R}{d t^{\prime}} d t^{\prime}=i \int_{R_{i}}^{R_{f}}\left\langle\varphi_{n}\left|\frac{\partial}{\partial R}\right| \varphi_{n}\right\rangle d R .
$$

Em particular, se o Hamiltoniano retorna ao estado inicial depois de um tempo $T$, de modo que $R_{f}=R_{i}$, então $\gamma_{n}(T)=0$, não surge nada diferente do usual.

Entretanto, na equação (3.2.2) estamos considerando que só um parâmetro no Hamiltoniano é modificado. Agora vamos supor que se tem $N$ parâmetros: $R_{1}(t), R_{2}(t), \ldots, R_{N}(t)$, então

$$
\frac{\partial \varphi_{n}}{\partial t}=\frac{\partial \varphi_{n}}{\partial R_{1}} \frac{d R_{1}}{d t}+\frac{\partial \varphi_{n}}{\partial R_{2}} \frac{d R_{2}}{d t}+\ldots+\frac{\partial \varphi_{n}}{\partial R_{N}} \frac{d R_{N}}{d t}=\left(\vec{\nabla}_{R} \varphi_{n}\right) \cdot \frac{d \vec{R}}{d t}
$$

Com isso a equação (3.2.2) fica

$$
\gamma_{n}(t)=i \int_{R_{i}}^{R_{f}}\left\langle\varphi_{n}\left|\vec{\nabla}_{R}\right| \varphi_{n}\right\rangle \cdot d \vec{R}
$$

Se o Hamiltoniano retorna ao estado inicial depois de um tempo $T$, a equação fica

$$
\gamma_{n}(T)=i \oint\left\langle\varphi_{n}\left|\vec{\nabla}_{R}\right| \varphi_{n}\right\rangle \cdot d \vec{R}
$$

Portanto, a fase $\gamma_{n}(t)$ depende somente do caminho fechado percorrido no espaço dos parâmetros que ocasionam a mudança do Hamiltoniano, não depende do tempo que o sistema demora em descrever a curva fechada $(5,32)$. Também podemos verificar que cada autoestado do Hamiltoniano tem sua própria fase geométrica. Uma propriedade da fase $\gamma_{n}$ é a sua invariância de gauge, o que significa dizer que não é possível removê-la através da simples adição de um fator de fase $\delta(\vec{R})$ ao estado. Para verificar tal propriedade, substituímos $\left|\varphi_{n}(\vec{R})\right\rangle$ por 
$\left|\varphi_{n}^{\prime}(\vec{R})\right\rangle=e^{i \delta(\vec{R})}\left|\varphi_{n}(\vec{R})\right\rangle$ da equação (3.2.4)

$$
\left\langle\varphi_{n}^{\prime}\left|\vec{\nabla}_{R}\right| \varphi_{n}^{\prime}\right\rangle=\left\langle\varphi_{n}\left|\vec{\nabla}_{R}\right| \varphi_{n}\right\rangle+i \vec{\nabla}_{R} \delta
$$

com isso

$$
\oint\left\langle\varphi_{n}^{\prime}\left|\vec{\nabla}_{R}\right| \varphi_{n}^{\prime}\right\rangle \cdot d \vec{R}=\oint\left\langle\varphi_{n}\left|\vec{\nabla}_{R}\right| \varphi_{n}\right\rangle \cdot d \vec{R}+i \oint \vec{\nabla}_{R} \delta \cdot d \vec{R}
$$

do Teorema de Stokes o ultimo termo é zero, então $\gamma_{n}^{\prime}(\vec{R})=\gamma_{n}(\vec{R})$.

\subsection{A condição para a adiabaticidade em um sistema fe- chado}

Vamos considerar um sistema quântico evoluindo seguindo a equação de Schrödinger (3.1.1). No momento inicial $t=0$, o sistema encontra-se no estado $|\psi(0)\rangle=\left|\varphi_{n}(0)\right\rangle$. Então para um tempo $t$, o sistema é descrito por

$$
|\psi(t)\rangle=\sum_{m} c_{m}\left|\varphi_{m}\right\rangle
$$

onde $c_{m}(t)=\left\langle\varphi_{m} \mid \psi(t)\right\rangle$ são os coeficientes dependentes do tempo. Supondo que o Hamiltoniano muda lentamente como o tempo, então do teorema adiabático obtemos

$$
\left|\psi^{a d i}(t)\right\rangle=e^{i \alpha(t)}\left|\varphi_{n}(t)\right\rangle
$$

onde $\alpha(t)$ é a fase total, ou seja, a soma da fase geométrica e dinâmica. Em geral, $\left|\psi^{\text {adi }}(t)\right\rangle$ não é uma solução da equação de Schrödinger. No entanto, para alguns sistemas quânticos, os Hamiltonianos com evolução lenta podem aproximadamente satisfazer a equação de Schrödinger (3.1.1)

$$
i \frac{d}{d t}\left|\psi^{a d i}(t)\right\rangle \approx H(t)\left|\psi^{a d i}(t)\right\rangle .
$$

Então $|\psi(t)\rangle \approx\left|\psi^{\text {adi }}\right\rangle$, também se verifica que $|\dot{\psi}(t)\rangle \approx\left|\dot{\psi}^{\text {adi }}\right\rangle$.

A validade da aproximação adiabática implica que

$$
\left|c_{m}(t)\right|=\left|\left\langle\varphi_{m}(t) \mid \psi(t)\right\rangle\right| \ll 1, m \neq n
$$


Porém, sendo $\left\langle\varphi_{m}\left|H(t)-E_{n}\right| \psi(t)\right\rangle=\left(E_{m}-E_{n}\right)\left\langle\varphi_{m} \mid \psi(t)\right\rangle$, os coeficientes $c_{m}(t)$ ficam

$$
c_{m}(t)=\left\langle\varphi_{m} \mid \psi\right\rangle=\frac{1}{E_{m}-E_{n}}\left\langle\varphi_{m}\left|H(t)-E_{n}\right| \psi(t)\right\rangle .
$$

Da equação de Schrödinger, $H(t)|\psi(t)\rangle=i|\dot{\psi}(t)\rangle$, a equação (3.3.5) pode ser escrita como

$$
\begin{aligned}
c_{m}(t) & =\frac{1}{E_{m}-E_{n}}\left\langle\varphi _ { m } \left|\left(i|\dot{\psi}\rangle-E_{n}|\psi\rangle\right)\right.\right. \\
c_{m}(t) & \approx \frac{1}{E_{m}-E_{n}}\left\langle\varphi _ { m } \left|\left(i\left|\dot{\psi}^{a d i}\right\rangle-E_{n}\left|\psi^{a d i}\right\rangle\right)\right.\right. \\
c_{m}(t) & =\frac{e^{i \alpha}}{E_{m}-E_{n}}\left\langle\varphi _ { m } \left|\left(i\left|\dot{\varphi}_{n}\right\rangle-\left(\dot{\alpha}+E_{n}\right)\left|\varphi_{n}\right\rangle\right)\right.\right. \\
c_{m}(t) & =i e^{i \alpha} \frac{\left\langle\varphi_{m} \mid \dot{\varphi}_{n}\right\rangle}{E_{m}-E_{n}}
\end{aligned}
$$

O resultado anterior mostra que a aproximação adiabática é valida para o sistema quando $c_{m}(t)$ é aproximadamente igual a $\left\langle\varphi_{m} \mid \dot{\varphi}_{n}\right\rangle / E_{m}-E_{n}$ menos um fator de fase. Logo, da equação (3.3.4) temos a condição que um sistema que evolui adiabaticamente $(33,34)$ deve satisfazer

$$
\left|\frac{\left\langle\varphi_{m}|\dot{H}| \varphi_{n}\right\rangle}{\left(E_{m}-E_{n}\right)^{2}}\right|=\left|\frac{\left\langle\varphi_{m} \mid \dot{\varphi}_{n}\right\rangle}{E_{m}-E_{n}}\right| \ll 1, m \neq n
$$

\subsection{Exemplo de fase geométrica em uma partícula de spin}

Vamos supor uma partícula de spin $s=1 / 2$, na presença de um campo magnético constante cuja magnitude é $B_{0}$, mas a direção varre um cone com ângulo de abertura $\alpha$, a uma velocidade angular constante $(2,33)$, como se mostra na figura (3.1). Seja o campo magnético

$$
\vec{B}(t)=B_{0}[\operatorname{sen} \alpha \cos \omega t \hat{i}+\operatorname{sen} \alpha \operatorname{sen} \omega t \hat{j}+\cos \alpha \hat{k}]
$$

o Hamiltoniano que representa o acomplamento spin-campo é

$$
H(t)=\hbar \gamma \vec{B} \cdot \vec{S}
$$




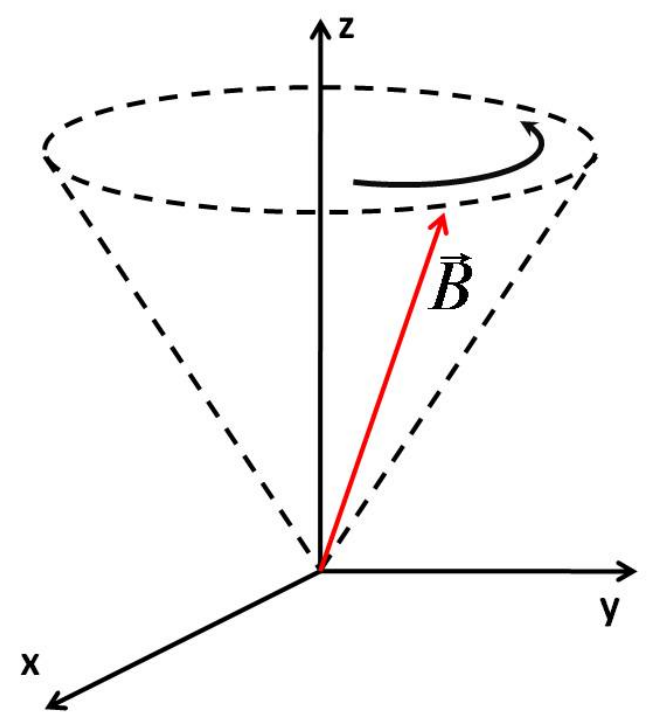

Figura 3.1-Campo Magnético girando em torno de $z$.

Fonte: Elaborada pelo autor.

ou na forma matricial

$$
H(t)=\frac{\hbar \omega_{1}}{2}\left(\begin{array}{rr}
\cos \alpha & e^{-i \omega t} \operatorname{sen} \alpha \\
e^{i \omega t} \operatorname{sen} \alpha & -\cos \alpha
\end{array}\right)
$$

onde $\gamma$ é o fator giromagnético e $\omega_{1}=\gamma B_{0}$. Podemos encontrar os autovetores e autovalores do Hamiltoniano

$$
\begin{gathered}
\left|\varphi_{+}(t)\right\rangle=\left(\begin{array}{r}
\cos \alpha / 2 \\
e^{i \omega t} \operatorname{sen} \alpha / 2
\end{array}\right) ;\left|\varphi_{-}(t)\right\rangle=\left(\begin{array}{r}
e^{-i \omega t} \operatorname{sen} \alpha / 2 \\
-\cos \alpha / 2
\end{array}\right) \\
E_{ \pm}= \pm \frac{\hbar \omega_{1}}{2}
\end{gathered}
$$

Vamos considerar que o estado inicial do elétron seja $\left|\varphi_{+}(0)\right\rangle=\left(\begin{array}{c}\cos \alpha / 2 \\ \operatorname{sen} \alpha / 2\end{array}\right)$. Resolvendo a equação de Schrödinger

$$
i \hbar \frac{d|\psi(t)\rangle}{d t}=H(t)|\psi(t)\rangle
$$

tal que $|\psi(t)\rangle=\left(\begin{array}{l}a \\ b\end{array}\right)$, temos o seguinte sistema de equações

$$
\frac{d a}{d t}=-\frac{i \omega_{1}}{2}\left(a \cos \alpha+b \operatorname{sen} \alpha e^{-i \omega t}\right)
$$




$$
\frac{d b}{d t}=-\frac{i \omega_{1}}{2}\left(a e^{i \omega t} \operatorname{sen} \alpha-b \cos \alpha\right)
$$

cuja solução será

$$
|\psi(t)\rangle=\left(\begin{array}{r}
{\left[\cos \left(\frac{\lambda t}{2}\right)-i\left(\frac{\omega_{1}-\omega}{\lambda}\right)\right] \cos \left(\frac{\alpha}{2}\right) e^{-i \omega t / 2}} \\
{\left[\cos \left(\frac{\lambda t}{2}\right)-i\left(\frac{\omega_{1}+\omega}{\lambda}\right)\right] \cos \left(\frac{\alpha}{2}\right) e^{i \omega t / 2}}
\end{array}\right)
$$

$\operatorname{com} \lambda=\sqrt{\omega_{1}^{2}+\omega^{2}-2 \omega \omega_{1} \cos \alpha}$. O autoestado final se pode pôr em função dos autovetores do Hamiltoniano

$$
\begin{aligned}
|\psi(t)\rangle & =\left[\cos \left(\frac{\lambda t}{2}\right)-i\left(\frac{\omega_{1}-\omega}{\lambda}\right) \operatorname{sen}\left(\frac{\lambda t}{2}\right)\right] e^{-i \omega t / 2}\left|\varphi_{+}(t)\right\rangle \\
& + \\
& i\left[\frac{\omega}{\lambda} \operatorname{sen} \alpha \operatorname{sen}\left(\frac{\lambda t}{2}\right)\right] e^{i \omega t / 2}\left|\varphi_{-}(t)\right\rangle
\end{aligned}
$$

De onde podemos ver que a probabilidade de transição ao spin down é

$$
\left|\left\langle\psi(t) \mid \varphi_{-}(t)\right\rangle\right|^{2}=\left[\frac{\omega}{\lambda} \operatorname{sen} \alpha \operatorname{sen}\left(\frac{\lambda t}{2}\right)\right]^{2}
$$

O teorema adiabático diz que essa probabilidade de transição deve desaparecer quando o campo gira lentamente em comparação com a fase da função de onda $\left(\omega \ll \omega_{1}\right)$, então

$$
\left|\left\langle\psi(t) \mid \varphi_{-}(t)\right\rangle\right|^{2} \approx\left[\frac{\omega}{\omega_{1}} \operatorname{sen} \alpha \operatorname{sen}\left(\frac{\lambda t}{2}\right)\right]^{2} \rightarrow 0
$$

Logo

$$
|\psi(t)\rangle=\left[\cos \left(\frac{\lambda t}{2}\right)-i\left(\frac{\omega_{1}-\omega}{\lambda}\right) \operatorname{sen}\left(\frac{\lambda t}{2}\right)\right] e^{-i \omega t / 2}\left|\varphi_{+}(t)\right\rangle .
$$

Na aproximação adiabática $\lambda=\omega_{1}-\omega \cos \alpha$. Assim

$$
|\psi(t)\rangle=e^{-i \omega t / 2} e^{-i \omega_{1} t / 2} e^{i \omega \cos \alpha t / 2}\left|\varphi_{+}(t)\right\rangle
$$

Usando a equação (3.2.1) encontramos a fase dinâmica

$$
\theta_{+}(T)=\frac{1}{\hbar} \int_{0}^{T} \frac{\hbar \omega_{1}}{2} \rightarrow \theta_{+}(t)=\frac{\omega_{1}}{2} T
$$

e para encontrar a fase geométrica usaremos a equação (3.2.2)

$$
\begin{gathered}
\gamma_{+}(T)=i \int_{0}^{T}\left(\begin{array}{ll}
\cos \alpha / 2 & e^{-i \omega t} \operatorname{sen} \alpha / 2
\end{array}\right)\left(\begin{array}{r}
0 \\
i \omega e^{i \omega t} \operatorname{sen} \alpha / 2
\end{array}\right) d t^{\prime} \\
\gamma_{+}(T)=(\cos \alpha-1) \frac{\omega T}{2}
\end{gathered}
$$

Então, as fases de Berry adquiridas pelos autoestados $\left|\varphi_{ \pm}\right\rangle$após do período $T=2 \pi / \omega$ é 


$$
\begin{gathered}
\gamma_{ \pm}(T)=\mp \pi(1-\cos \theta) \\
\gamma_{ \pm}(T)=\mp \frac{\Omega}{2}
\end{gathered}
$$

Sendo $\Omega$ o ângulo sólido englobados pelas trajetórias descritas pelos autoestados $\left|\varphi_{ \pm}\right\rangle$, a partir da origem do sistema de coordenandas. Desta última expressão, conclui-se que a fase de Berry depende somente da área descrita pelo vetor $R(\vec{t})$ no espaço de parâmetros.

\subsection{Aproximação adiabática em sistemas abertos}

Nesta seção, vamos a introduzir o teorema adiabático para o caso de sistemas quânticos abertos (34). Vamos considerar um sistema quântico $S$ acoplado a um ambiente, ou banho $B$, evoluindo unitariamente sobre o Hamiltoniano do sistema total. A dinâmica exata do sistema é dada pelo rastreamento sobre os graus de liberdade do banho (10).

$$
\rho(t)=\operatorname{Tr}_{B}\left[U(t) \rho_{S B}(0) U^{\dagger}(t)\right]
$$

onde $\rho(t)$ é o operador densidade do sistema, $\rho_{S B}=\rho(0) \otimes \rho_{B}(0)$ é o estado do sistema-banho no inicio, e $U(t)$ é o operador evolução. Considerando o regime Markoviano a equação (3.5.1) pode-se expressar assim

$$
\dot{\rho}(t)=\mathcal{L}(t) \rho(t) .
$$

Um exemplo importante é

$$
\dot{\rho}(t)=-i[H(t), \rho(t)]+\frac{1}{2} \sum_{i=1}^{N}\left(\left[\Gamma_{i}(t), \rho(t) \Gamma_{i}^{\dagger}\right]+\left[\Gamma_{i}(t) \rho(t), \Gamma_{i}^{\dagger}\right]\right)
$$

onde $H(t)$ é o Hamiltoniano efetivo dependente do tempo do sistema de interesse e $\Gamma_{i}(t)$ são operadores dependentes do tempo que descrevem a interação do sistema-banho. Abusando da nomenclatura, vamos usar a partir de agora $\mathcal{L}(t)$ como superoperador Lindblad e a $\Gamma_{i}(t)$ como operadores Lindblad. A dificuldade na transição de uma aproximação adiabática de um sistema quântico fechado para um sistema quântico aberto é que a noção de autoestados do Hamiltoniano se perde, já que o superoperador de Lindblad não pode ser diagonalizado em geral. Então não é fácil saber quem deve tomar o lugar dos autoestados adiabáticos. Contudo, esta dificuldade foi resolvida na referencia (11), introduzindo o formalismo matemático para obter os blocos de Jordan com seus autovetores direita e esquerda. Neste sentido, é conveni- 
ente trabalhar no formalismo do superoperador, onde a matriz densidade é representada numa base formada por um conjunto ortonormal de matrizes $F_{i}(9)$, que satisfazem as seguintes condições $F_{i}=F_{i}^{\dagger}, \operatorname{Tr}\left(F_{i}\right)=0$ e $\operatorname{Tr}\left(F_{i} F_{k}\right)=\delta_{i k}$. De forma geral temos

$$
|\rho\rangle\rangle=\left(\rho_{1}, \rho_{2}, \ldots, \rho_{D^{2}}\right)^{t}
$$

onde $\rho_{i}$ são os coeficientes de expansão de $\rho$. Usamos a notação de bracket duplo para indicar que não estamos trabalhando no espaço de Hilbert tradicional. O vetor $|\rho\rangle\rangle$, conhecido como vetor de coerência, vive no espaço de Hilber-Schmidt: um espaço onde os operadores lineares estão dotados com um produto interno que pode ser definido para os vetores $|u\rangle\rangle$ e $\langle\langle v|$ como

$$
\langle\langle v \mid u\rangle\rangle=\frac{1}{N} \operatorname{Tr}\left(v^{\dagger} u\right)
$$

sendo $N$ o fator de normalização. Podemos reescrever a equação (3.5.2), como

$$
\mathcal{L}(t)|\rho(t)\rangle\rangle=|\dot{\rho}(t)\rangle\rangle
$$

Esta equação mestra gera uma evolução não unitária, já que $\mathcal{L}(t)$ não é Hermitiana e geralmente não diagonalizável. No entanto, é sempre possível transformar $\mathcal{L}(t)$ na forma canônica de Jordan (35), onde ele tem uma estrutura de bloco diagonal. Isto é conseguido mediante transformações de similaridade.

$$
\mathcal{L}_{J}(t)=S^{-1}(t) \mathcal{L}(t) S(t)
$$

onde $\mathcal{L}_{J}(t)=\operatorname{diag}\left(J_{1}, \ldots, J_{m}\right)$ denota a forma de Jordan de $\mathcal{L}(t)$. Os blocos de Jordan $J_{\alpha}$, de dimensão $n_{\alpha}$, são sempre da forma

$$
J_{\alpha}=\left(\begin{array}{ccccc}
\lambda_{\alpha} & 1 & 0 & \cdots & 0 \\
0 & \lambda_{\alpha} & 1 & \cdots & 0 \\
\vdots & & \ddots & & \vdots \\
0 & \cdots & \cdots & \lambda_{\alpha} & 1 \\
0 & \cdots & \cdots & 0 & \lambda_{\alpha}
\end{array}\right)
$$

Para cada bloco de Jordan está associado um autovetor direito e um autovetor esquerdo com autovalor $\lambda_{\alpha}$, que em geral pode ser complexo $(9,11)$. O número $m$ de blocos de Jordan é dada pelo número de autoestados linearmente independentes de $\mathcal{L}(t)$, com cada autoestado associado a um bloco $J_{\alpha}$ diferente. Como $\mathcal{L}(t)$ não é Hermitiana, geralmente não temos uma base de autoestados, daí que é necessário algum cuidado, a fim de encontrar uma base para a descrição do operador densidade. Na referência (11) é mostrado que as bases instantâneas $\left.\left\{\left|D_{\beta}^{(j)}\right\rangle\right\rangle\right\}$ e $\left\{\left\langle\left\langle E_{\alpha}^{(i)}\right|\right\}\right.$ no espaço de estados dos operadores lineares podem sempre ser 
sistematicamente construídas com as seguintes características:

- Condição de ortonormalidade:

$$
\left\langle\left\langle E_{\alpha}^{(i)} \mid D_{\beta}^{(j)}\right\rangle\right\rangle=\delta_{\alpha \beta} \delta_{i j}
$$

- Invariância dos blocos de Jordan sobre a ação do superoperador Lindblad:

$$
\begin{gathered}
\left.\left.\left.\mathcal{L}(t)\left|D_{\alpha}^{(j)}\right\rangle\right\rangle=\left|D_{\alpha}^{(j-1)}\right\rangle\right\rangle+\lambda_{\alpha}(t)\left|D_{\alpha}^{(j)}\right\rangle\right\rangle \\
\left\langle\left\langle E_{\alpha}^{(i)}\right| \mathcal{L}(t)=\left\langle\left\langle E_{\alpha}^{(i+1)}\right|+\left\langle\left\langle E_{\alpha}^{(i)}\right| \lambda_{\alpha}(t)\right.\right.\right.
\end{gathered}
$$

$\left.\operatorname{com}\left|D_{\alpha}^{(-1)}\right\rangle\right\rangle=0$ e $\left\langle\left\langle E_{\alpha}^{\left(n_{\alpha}\right)}\right|=0\right.$. Os índices inferiores enumeram os blocos de Jordan $(\alpha \in\{1, \ldots, m\})$, enquanto os índices superiores enumeram os estados da base dentro de um determinado bloco de Jordan $\left(i, j \in 0, \ldots, n_{\alpha}-1\right)$.

Antes de apresentar explicitamente as condições para a evolução adiabática, fornecemos uma definição formal da adiabaticidade para o caso de sistemas abertos:

Definição $(11,36)$ : Um sistema quântico aberto segue uma dinâmica adiabática se o seu espaço de Hilbert-Schmidt pode ser descomposto em auto-espaços Lindblad-Jordan desacoplados com diferentes autovalores instantâneos, não cruzados, de $\mathcal{L}(t)$.

Esta definição é uma extensão natural para os sistemas abertos da idéia de comportamento adiabático. A divisão em blocos de Jordan do superoperador Lindblad é conseguido através da escolha de uma base que preserva a estrutura de bloco de Jordan como, por exemplo, o conjunto $\left.\left\{\left|D_{\beta}^{(j)}\right\rangle\right\rangle\right\}$ e $\left\{\left\langle\left\langle E_{\alpha}^{(i)}\right|\right\}\right.$.

Vamos agora a derivar as condições de validade para a dinâmica adiabática para sistemas abertos, analisando a evolução do operador densidade sobre a equação mestra (3.5.6). Para este fim, vamos expandir a matriz densidade para um tempo arbitrário $t$ na base de autoestados instantâneos $\left.\left\{\left|D_{\beta}^{(j)}(t)\right\rangle\right\rangle\right\}$, então

$$
\left.|\rho(t)\rangle\rangle=\frac{1}{2} \sum_{\beta=1}^{m} \sum_{j=0}^{n_{\beta}-1} r_{\beta}^{(j)}(t)\left|D_{\beta}^{(j)}(t)\right\rangle\right\rangle
$$

onde $m$ é o número de blocos de Jordan e $n_{\beta}$ é a dimensão do bloco $J_{\beta}$. Ressaltamos que estamos supondo que não há cruzamentos do autovalor no espectro do superoperador Lindblad durante a evolução. Agora vamos a exigir que o operador densidade (3.5.12) evolua sobre a 
equação mestra (3.5.6) e fazendo uso da equação (3.5.10), obtemos

$$
\left.\left.\sum_{\beta=1}^{m} \sum_{j=0}^{n_{\beta}-1} r_{\beta}^{(j)}\left(\left|D_{\beta}^{(j-1)}(t)\right\rangle\right\rangle+\lambda_{\beta}(t)\left|D_{\beta}^{(j)}(t)\right\rangle\right\rangle\right)
$$

multiplicando por $\left\langle\left\langle E_{\alpha}^{(i)}\right|\right.$ obtemos

$$
\dot{r}_{\alpha}^{(i)}=\lambda_{\alpha} r^{(i+1)} \alpha-\sum_{\beta=1}^{m} \sum_{j=0}^{n_{\beta}-1} r_{\beta}^{(j)}\left\langle\left\langle E_{\alpha}^{(i)} \mid \dot{D}_{\beta}^{(j)}\right\rangle\right\rangle
$$

$\operatorname{com} r_{\alpha}^{\left(n_{\alpha}\right)}(t)=0$. Note que a soma sobre $\beta$ mistura diferentes blocos de Jordan. Agora devemos separar esta soma em termos relacionados ao autovalor $\lambda_{\alpha}$ de $\mathcal{L}(t)$ e termos que envolvem a mistura com autovalores $\lambda_{\beta} \neq \lambda_{\alpha}$. Neste último caso, a expressão pode ser encontrada por $\left\langle\left\langle E_{\alpha}^{(i)} \mid \dot{D}_{\beta}^{(j)}\right\rangle\right\rangle$ como se segue:

$$
\left.\left.\left.\mathcal{L}(t)\left|D_{\beta}^{(j)}\right\rangle\right\rangle=\left|D_{\beta}^{(j-1)}\right\rangle\right\rangle+\lambda_{\beta}(t)\left|D_{\beta}^{(j)}\right\rangle\right\rangle
$$

derivando encontramos

$$
\left.\left.\left.\left.\left.\dot{\mathcal{L}}(t)\left|D_{\beta}^{(j)}\right\rangle\right\rangle+\mathcal{L}(t)\left|\dot{D}_{\beta}^{(j)}\right\rangle\right\rangle=\left|\dot{D}_{\beta}^{(j-1)}\right\rangle\right\rangle+\dot{\lambda}_{\beta}(t)\left|D_{\beta}^{(j)}\right\rangle\right\rangle+\lambda_{\beta}(t)\left|\dot{D}_{\beta}^{(j)}\right\rangle\right\rangle
$$

multiplicando por $\left\langle\left\langle E_{\alpha}^{(i)}\right|\right.$, obtemos

$$
\left\langle\left\langle E_{\alpha}^{(i)} \mid \dot{D}_{\beta}^{(j)}\right\rangle\right\rangle=\frac{1}{\omega_{\beta \alpha}}\left[\left\langle\left\langle E_{\alpha}^{(i)}|\dot{\mathcal{L}}(t)| D_{\beta}^{(j)}\right\rangle\right\rangle+\left\langle\left\langle E_{\alpha}^{(i+1)} \mid \dot{D}_{\beta}^{(j)}\right\rangle\right\rangle-\left\langle\left\langle E_{\alpha}^{(i)} \mid \dot{D}_{\beta}^{(j-1)}\right\rangle\right\rangle\right], \lambda_{\alpha} \neq \lambda_{\beta}
$$

onde definimos $\omega_{\beta \alpha}=\lambda_{\beta}-\lambda_{\alpha}$. A partir da iteração recursiva do último termo, obtemos

$$
\left\langle\left\langle E_{\alpha}^{(i)} \mid \dot{D}_{\beta}^{(j)}\right\rangle\right\rangle=\sum_{k=0}^{j} \frac{(-1)^{k}}{\omega_{\beta \alpha}^{k+1}}\left(\left\langle\left\langle E_{\alpha}^{(i)}|\dot{\mathcal{L}}| D_{\beta}^{(j-k)}\right\rangle\right\rangle+\left\langle\left\langle E_{\alpha}^{(i+1)} \mid \dot{D}_{\beta}^{(j-k)}\right\rangle\right\rangle\right)
$$

Seguindo um procedimento semelhante pode-se obter todos os termos $\left\langle\left\langle E_{\alpha}^{(i)} \mid \dot{D}_{\beta}^{(j-k)}\right\rangle\right\rangle$, então a equação anterior fica assim

$$
\left\langle\left\langle E_{\alpha}^{(i)} \mid \dot{D}_{\beta}^{(j)}\right\rangle\right\rangle=\sum_{p=1}^{n_{\alpha}-1}\left(\prod_{q=1}^{p} \sum_{k_{q}=0}^{j-S_{q-1}}\right) \frac{\left\langle\left\langle E_{\alpha}^{(i-p-1)}|\dot{\mathcal{L}}| D_{\beta}^{\left(j-S_{p}\right)}\right\rangle\right\rangle}{(-1)^{S_{p}} \omega_{\beta \alpha}^{p+S_{p}}}
$$

onde

$$
S_{q}=\sum_{s=1}^{q} k_{s}, S_{0}=0
$$


e

$$
\left(\prod_{q=1}^{p} \sum_{k_{q}=0}^{j-S_{q-1}}\right) \equiv \sum_{k_{1}=0}^{j-S_{0}} \cdots \sum_{k_{p}=0}^{j-S_{p-1}}
$$

Da equação (3.5.14)

$$
\dot{r}_{\alpha}^{(i)}=\lambda_{\alpha} r^{(i)} \alpha+r_{\alpha}^{(i+1)}-\sum_{\beta \mid \lambda_{\beta}=\lambda_{\alpha}}^{m} \sum_{j=0}^{n_{\beta}-1} r_{\beta}^{(j)}\left\langle\left\langle E_{\alpha}^{(i)} \mid \dot{D}_{\beta}^{(j)}\right\rangle\right\rangle-\sum_{\beta \mid \lambda_{\beta} \neq \lambda_{\alpha}}^{m} \sum_{j=0}^{n_{\beta}-1} r_{\beta}^{(j)}\left\langle\left\langle E_{\alpha}^{(i)} \mid \dot{D}_{\beta}^{(j)}\right\rangle\right\rangle
$$

como no caso do sistema fechado, o último termo da equação anterior deve ser desprezível, então a condição para a aproximação adiabática no sistema aberto é

$$
\sum_{p=1}^{n_{\alpha}-1}\left(\prod_{q=1}^{p} \sum_{k_{q}=0}^{j-S_{q-1}}\right) \frac{\left\langle\left\langle E_{\alpha}^{(i-p-1)}|\dot{\mathcal{L}}| D_{\beta}^{\left(j-S_{p}\right)}\right\rangle\right\rangle}{(-1)^{S_{p}} \omega_{\beta \alpha}^{p+S_{p}}} \ll 1, \lambda_{\beta} \neq \lambda_{\alpha} .
$$

\subsection{Blocos de Jordan de uma dimensão}

Vamos a considerar o caso particular onde $\mathcal{L}(t)$ tem apenas uma dimensão e cada autovalor corresponde a um único autovetor independente, isto é, $\lambda_{\alpha}=\lambda_{\beta} \Rightarrow \alpha=\beta$. Tendo em conta essas condições a equação (3.5.14) pode ser reescrita como

$$
\dot{r}_{\alpha}=\lambda_{\alpha} r_{\alpha}-r_{\alpha}\left\langle\left\langle E_{\alpha} \mid \dot{D}_{\alpha}\right\rangle\right\rangle-\sum_{\beta \neq \alpha} r_{\beta}\left\langle\left\langle E_{\alpha} \mid \dot{D}_{\beta}\right\rangle\right\rangle
$$

Além disso, para este caso especial a equação (3.5.17) fica da seguinte forma

$$
\left\langle\left\langle E_{\alpha} \mid \dot{D}_{\beta}\right\rangle\right\rangle=\frac{\left\langle\left\langle E_{\alpha}|\dot{\mathcal{L}}| D_{\beta}\right\rangle\right\rangle}{\omega_{\beta \alpha}}
$$

A fim de eliminar o termo $\lambda_{\alpha} r_{\alpha}$ da equação (3.6.1), definimos a variável $r_{\alpha}(t)$ como

$$
r_{\alpha}(t)=p_{\alpha} \exp \left(\int_{0}^{t} \lambda_{\alpha}\left(t^{\prime}\right) d t^{\prime}\right)
$$

substituindo na equação (3.6.1), obtemos a seguinte equação

$$
\dot{p}_{\alpha}=-p_{\alpha}\left\langle\left\langle E_{\alpha} \mid \dot{D}_{\alpha}\right\rangle\right\rangle-\sum_{\beta \neq \alpha} p_{\beta}\left\langle\left\langle E_{\alpha} \mid \dot{D}_{\beta}\right\rangle\right\rangle e^{\Omega_{\beta \alpha}}
$$

sendo

$$
\Omega_{\beta \alpha}(t)=\int_{0}^{t} \omega_{\beta \alpha}\left(t^{\prime}\right) .
$$


A equação (3.6.4) é muito semelhante à equação (3.1.9) para sistemas fechados, mas o fato de que $\Omega_{\beta \alpha}$ seja complexo em geral, leva a algumas diferenças. Agora vamos a introduzir a variável $s=t / T$, tal que $0 \leq s \leq 1$, substituindo na equação anterior e integrando obtemos

$$
p_{\alpha}(s)=p_{\alpha}(0)-\int_{0}^{s} d s^{\prime} p_{\alpha}\left(s^{\prime}\right) \Phi_{\alpha}\left(s^{\prime}\right)-\sum_{\beta \neq \alpha} \int_{0}^{s} d s^{\prime} \frac{V_{\beta \alpha}\left(s^{\prime}\right)}{\omega_{\beta \alpha}\left(s^{\prime}\right)} e^{T \Omega_{\beta \alpha}\left(s^{\prime}\right)}
$$

onde

$$
\Phi_{\alpha}(s)=\left\langle\left\langle E_{\alpha}(s)\left|\frac{d}{d s}\right| D_{\alpha}(s)\right\rangle\right\rangle
$$

e

$$
V_{\beta \alpha}(s)=p_{\beta}(s)\left\langle\left\langle E_{\alpha}(s)\left|\frac{d \mathcal{L}(s)}{d s}\right| D_{\beta}(s)\right\rangle\right\rangle .
$$

O ultimo integrando da equação (3.6.5), pode se reescrever como

$$
\frac{V_{\beta \alpha}(s)}{\omega_{\beta \alpha}(s)} e^{T \Omega_{\beta \alpha}(s)}=\frac{1}{T}\left[\frac{d}{d s}\left(\frac{V_{\beta \alpha}(s)}{\omega_{\beta \alpha}^{2}(s)} e^{T \Omega_{\beta \alpha}(s)}\right)-e^{T \Omega_{\beta \alpha}(s)} \frac{d}{d s} \frac{V_{\beta \alpha}(s)}{\omega_{\beta \alpha}^{2}(s)}\right]
$$

Então substituindo na equação (3.6.5), temos

$$
\begin{array}{r}
p_{\alpha}(s)=p_{\alpha}(0)-\int_{0}^{s} d s^{\prime} p_{\alpha}\left(s^{\prime}\right) \Phi_{\alpha}\left(s^{\prime}\right)+ \\
+\frac{1}{T} \sum_{\beta \neq \alpha}\left(\frac{V_{\beta \alpha}(0)}{\omega_{\beta \alpha}^{2}(0)}-\left(\frac{V_{\beta \alpha}(s)}{\omega_{\beta \alpha}^{2}(s)} e^{T \Omega_{\beta \alpha}(s)}+\int_{0}^{s} d s^{\prime} e^{T \Omega_{\beta \alpha}\left(s^{\prime}\right)} \frac{d}{d s^{\prime}} \frac{V_{\beta \alpha}\left(s^{\prime}\right)}{\omega_{\beta \alpha}^{2}\left(s^{\prime}\right)}\right) .\right.
\end{array}
$$

Assim a condição de adiabaticidade em termos do tempo total de evolução pode ser dado comparando $T$ com os termos que envolvem os índices $\beta \neq \alpha$,ou seja

$$
\max _{0 \leq s \leq 1} \mid \sum_{\beta \neq \alpha}\left(\frac{V_{\beta \alpha}(0)}{\omega_{\beta \alpha}^{2}(0)}-\left(\frac{V_{\beta \alpha}(s)}{\omega_{\beta \alpha}^{2}(s)} e^{T \Omega_{\beta \alpha}(s)}+\int_{0}^{s} d s^{\prime} e^{T \Omega_{\beta \alpha}\left(s^{\prime}\right)} \frac{d}{d s^{\prime}} \frac{V_{\beta \alpha}\left(s^{\prime}\right)}{\omega_{\beta \alpha}^{2}\left(s^{\prime}\right)}\right) \mid \ll T .\right.
$$

\subsection{Exemplo: A evolução adiabática de um sistema aberto quântico de dois níveis}

Suponhamos que um sistema quântico de dois níveis com Hamiltoniano interno $H(t)=$ $\frac{\omega_{0}}{2} \sigma_{z}$ e submetido a uma fonte de decoerência $\Gamma(t)=\sum \alpha_{i}(t) \sigma_{i}$ é descrita pela equação 
(3.5.3)

$$
\dot{\rho(t)}=-i[H(t), \rho(t)]-\frac{1}{2}\left[\Gamma^{\dagger} \Gamma(t) \rho(t)+\rho(t) \Gamma^{\dagger} \Gamma(t)-2 \Gamma(t) \rho(t) \Gamma^{\dagger}\right] .
$$

Escrevendo o operador densidade na base $\left\{I, \sigma_{x}, \sigma_{y}, \sigma_{z}\right\}$, ou seja, como $\rho=(I+\vec{v} \cdot \vec{\sigma}) / 2$ obtemos

$$
\dot{\rho(t)}=\frac{\omega_{0}}{2}\left(v_{x} \sigma_{y}-v_{y} \sigma_{x}\right)+\sum_{i j} \alpha_{i}^{*} \alpha_{j}\left(v_{i} \sigma_{j}+v_{j} \sigma_{i}\right)-\sum_{i k}\left|\alpha_{i}\right| v_{k} \sigma_{k}-i \sum_{i j k} \epsilon_{i j k} \alpha_{i}^{*} \alpha_{j} \sigma_{k}
$$

expandindo as somatórias a equação anterior fica

$$
\begin{aligned}
\dot{\rho(t)} & =\left[-\frac{\omega_{0} v_{y}}{2}-\left(\left|\alpha_{y}\right|^{2}+\left|\alpha_{z}\right|^{2}\right) v_{x}+\left(\alpha_{x}^{*} \alpha_{y}+\alpha_{y}^{*} \alpha_{x}\right) \frac{v_{y}}{2}+\left(\alpha_{x}^{*} \alpha_{z}+\alpha_{z}^{*} \alpha_{x}\right) \frac{v_{z}}{2}-i\left(\alpha_{y}^{*} \alpha_{z}-\alpha_{z}^{*} \alpha_{y}\right)\right] \sigma_{x}+ \\
& +\left[\frac{\omega_{0} v_{x}}{2}-\left(\left|\alpha_{x}\right|^{2}+\left|\alpha_{z}\right|^{2}\right) v_{y}-\left(\alpha_{x}^{*} \alpha_{y}+\alpha_{y}^{*} \alpha_{x}\right) \frac{v_{x}}{2}+\left(\alpha_{y}^{*} \alpha_{z}+\alpha_{z}^{*} \alpha_{y}\right) \frac{v_{z}}{2}+i\left(\alpha_{x}^{*} \alpha_{z}-\alpha_{z}^{*} \alpha_{x}\right)\right] \sigma_{y}+ \\
& +\left[-\left(\left|\alpha_{x}\right|^{2}+\left|\alpha_{y}\right|^{2}\right) v_{z}+\left(\alpha_{x}^{*} \alpha_{z}+\alpha_{z}^{*} \alpha_{x}\right) \frac{v_{x}}{2}+\left(\alpha_{y}^{*} \alpha_{z}+\alpha_{z}^{*} \alpha_{y}\right) \frac{v_{y}}{2}-i\left(\alpha_{x}^{*} \alpha_{y}-\alpha_{y}^{*} \alpha_{x}\right)\right] \sigma_{z} .
\end{aligned}
$$

No formalismo do superoperador

$$
\mathcal{L}|\rho\rangle\rangle=|\dot{\rho}\rangle\rangle
$$

$\mathcal{L}\left(\begin{array}{c}1 \\ v_{x} \\ v_{y} \\ v_{z}\end{array}\right)=2\left(\begin{array}{c}0 \\ -\frac{\omega_{0} v_{y}}{2}-\left(\left|\alpha_{y}\right|^{2}+\left|\alpha_{z}\right|^{2}\right) v_{x}+\left(\alpha_{x}^{*} \alpha_{y}+\alpha_{y}^{*} \alpha_{x}\right) \frac{v_{y}}{2}+\left(\alpha_{x}^{*} \alpha_{z}+\alpha_{z}^{*} \alpha_{x}\right) \frac{v_{z}}{2}-i\left(\alpha_{y}^{*} \alpha_{z}-\alpha_{z}^{*} \alpha_{y}\right) \\ \frac{\omega_{0} v_{x}}{2}-\left(\left|\alpha_{x}\right|^{2}+\left|\alpha_{z}\right|^{2}\right) v_{y}+\left(\alpha_{x}^{*} \alpha_{y}+\alpha_{y}^{*} \alpha_{x}\right) \frac{v_{x}}{2}+\left(\alpha_{y}^{*} \alpha_{z}+\alpha_{z}^{*} \alpha_{y}\right) \frac{v_{z}}{2}+i\left(\alpha_{x}^{*} \alpha_{z}-\alpha_{z}^{*} \alpha_{x}\right) \\ -\left(\left|\alpha_{x}\right|^{2}+\left|\alpha_{y}\right|^{2}\right) v_{z}+\left(\alpha_{x}^{*} \alpha_{z}+\alpha_{z}^{*} \alpha_{x}\right) \frac{v_{x}}{2}+\left(\alpha_{y}^{*} \alpha_{z}+\alpha_{z}^{*} \alpha_{y}\right) \frac{v_{y}}{2}-i\left(\alpha_{x}^{*} \alpha_{y}-\alpha_{y}^{*} \alpha_{x}\right)\end{array}\right)$

então

$$
\mathcal{L}=\left(\begin{array}{cccc}
0 & 0 & 0 & 0 \\
-2 i\left(\alpha_{y}^{*} \alpha_{z}-\alpha_{z}^{*} \alpha_{y}\right) & -2\left(\left|\alpha_{y}\right|^{2}+\left|\alpha_{z}\right|^{2}\right) & \left(\alpha_{x}^{*} \alpha_{y}+\alpha_{y}^{*} \alpha_{x}\right)-\omega_{0} & \left(\alpha_{x}^{*} \alpha_{z}+\alpha_{z}^{*} \alpha_{x}\right) \\
2 i\left(\alpha_{x}^{*} \alpha_{z}-\alpha_{z}^{*} \alpha_{x}\right) & \omega_{0}+\left(\alpha_{x}^{*} \alpha_{y}-\alpha_{y}^{*} \alpha_{x}\right) & -2\left(\left|\alpha_{x}\right|^{2}+\left|\alpha_{z}\right|^{2}\right) & \left(\alpha_{y}^{*} \alpha_{z}+\alpha_{z}^{*} \alpha_{y}\right) \\
-2 i\left(\alpha_{x}^{*} \alpha_{y}-\alpha_{y}^{*} \alpha_{x}\right) & \left(\alpha_{x}^{*} \alpha_{z}+\alpha_{z}^{*} \alpha_{x}\right) & \left(\alpha_{y}^{*} \alpha_{z}+\alpha_{z}^{*} \alpha_{y}\right) & -2\left(\left|\alpha_{x}\right|^{2}+\left|\alpha_{y}\right|^{2}\right)
\end{array}\right)
$$

Se agora consideramos

$$
H_{1}(t)=\frac{\omega_{1}}{2}\left(\sigma_{x} \cos \omega t+\omega_{y} \operatorname{sen} \omega t\right)
$$


pode-se mostrar que o super-operador $\mathcal{L}_{1}$ correspondente as contribuições de $H_{1}$ é

$$
\mathcal{L}_{1}=\left(\begin{array}{cccc}
0 & 0 & 0 & 0 \\
0 & 0 & 0 & \omega_{1} \operatorname{sen} \omega t \\
0 & 0 & 0 & -\omega_{1} \cos \omega t \\
0 & -\omega_{1} \operatorname{sen} \omega t & \omega_{1} \cos \omega t & 0
\end{array}\right)
$$

Agora se consideramos o caso especial onde $\Gamma=\epsilon \sigma_{-}$, e o Hamiltoniano

$$
H(t)=\frac{\omega_{0}}{2} \sigma_{z}+\frac{\omega_{0}}{2}\left(\sigma_{x} \cos \omega t+\omega_{y} \operatorname{sen} \omega t\right)
$$

então das equações (3.7.4) e (3.7.5) obtemos

$$
\mathcal{L}=\epsilon^{2}\left(\begin{array}{cccc}
0 & 0 & 0 & 0 \\
0 & -1 / 2 & -w & w \operatorname{sen} \omega t \\
0 & w & -1 / 2 & -\omega_{0} \cos \omega t \\
-1 & -w \operatorname{sen} \omega t & w \cos \omega t & -1
\end{array}\right)
$$

sendo $w=\omega_{0} / \epsilon^{2}$. Os autovalores da matriz (3.7.6) são

$$
\begin{gathered}
\lambda_{1}=0 \\
4 \lambda_{i}^{3}+8 \lambda_{i}^{2}+\left(8 w^{2}+5\right) \lambda_{i}+1+6 w^{2}=0 \quad i=2,3,4
\end{gathered}
$$

os autovetores direita e esquerda são para $\lambda_{1}$

$$
\left.\left|D_{1}\right\rangle\right\rangle=\frac{1}{\sqrt{1+6 w^{2}}}\left(\begin{array}{c}
6 w^{2}+1 \\
-2 w(\operatorname{sen} \omega t+2 w \cos \omega t) \\
-2 w(2 w \operatorname{sen} \omega t-\cos \omega t) \\
-\left(4 w^{2}+1\right)
\end{array}\right), \quad\left\langle\left\langle E_{1}\right|=\left(\begin{array}{llll}
1 & 0 & 0 & 0
\end{array}\right)\right.
$$

para $\lambda_{i}$

$$
\left.\left|D_{i}\right\rangle\right\rangle=\frac{1}{\sqrt{M_{i}}}\left(\begin{array}{c}
a_{i} \\
b_{i} \\
c_{i} \\
d_{i}
\end{array}\right), \quad\left\langle\left\langle E_{i}\right|=\frac{1}{\sqrt{M_{i}}}\left(\begin{array}{cccc}
A_{i} & B_{i} & C_{i} & D_{i}
\end{array}\right)\right.
$$


onde

$$
\begin{gathered}
a_{i}=0 \\
b_{i}=2 w^{2}\left(2 w \cos \omega t+\left(2 \lambda_{i}+1\right) \operatorname{sen} \omega t\right) \\
c_{i}=2 w^{2}\left(2 w \operatorname{sen} \omega t-\left(2 \lambda_{i}+1\right) \cos \omega t\right) \\
d_{i}=4 w^{2}+\left(2 \lambda_{i}+1\right)^{2} \\
A_{i}=\frac{\lambda_{i}\left(2 \lambda_{i}+1\right) w}{1+\lambda_{i}} \\
B_{i}=2 w \cos \omega t-\left(2 \lambda_{i}+1\right) \operatorname{sen} \omega t \\
C_{i}=2 w \operatorname{sen} \omega t+\left(2+\lambda_{i}\right) \cos \omega t \\
D_{i}=-\frac{\left(2 \lambda_{i}+1\right) w}{1+\lambda_{i}} \\
M_{i}=A_{i} a_{i}+B_{i} b_{i}+C_{i} c_{i}+D_{i} d_{i}=\frac{4 w^{3}-w\left(2 \lambda_{i}+1\right)^{2}\left(4 \lambda_{i}+3\right)}{1+\lambda_{i}}
\end{gathered}
$$

pode-se mostrar que os autovetores direita e esquerda satisfazem a seguinte relação $\left\langle\left\langle E_{j} \mid D_{i}\right\rangle\right\rangle=$ $\delta_{i j}$. A forma de Jordan de $\mathcal{L}$ pode ser escrita assim

$$
\mathcal{L}_{J}=\left(\begin{array}{cccc}
0 & 0 & 0 & 0 \\
0 & \lambda_{2} & 0 & 0 \\
0 & 0 & \lambda_{3} & 0 \\
0 & 0 & 0 & \lambda_{4}
\end{array}\right)
$$

sendo a matriz de transformação que conduz à forma de Jordan

$$
S(t)=\left(\begin{array}{cccc}
a_{1} & a_{2} & a_{3} & a_{4} \\
b_{1} & b_{2} & b_{3} & b_{4} \\
c_{1} & c_{2} & c_{3} & c_{4} \\
d_{1} & d_{2} & d_{3} & d_{4}
\end{array}\right)
$$

Se consideramos que os $\lambda_{i}$ são diferentes, que é o mais provável pela forma da equação cúbica, então seguinte relação

$$
\left\langle\left\langle E_{i} \mid \dot{D}_{j}\right\rangle\right\rangle=\frac{\left\langle\left\langle E_{i}|\dot{\mathcal{L}}| D_{j}\right\rangle\right\rangle}{\omega_{j i}}
$$


com a qual podemos encontrar a condição adiabática para nosso caso

$$
\begin{aligned}
& C_{i j}=\left|\frac{\left\langle\left\langle E_{i} \mid \dot{D}_{j}\right\rangle\right\rangle}{\lambda_{j}-\lambda_{i}}\right|=\left|\frac{8 w^{2} \omega\left(\lambda_{i}+\lambda_{j}+1\right)}{\sqrt{M_{i} \times M_{j}}\left(\lambda_{j}-\lambda_{i}\right)}\right| \ll 1 \quad ; i, j=1,2,3,4 ; i \neq j \\
& C_{1 j}=0
\end{aligned}
$$

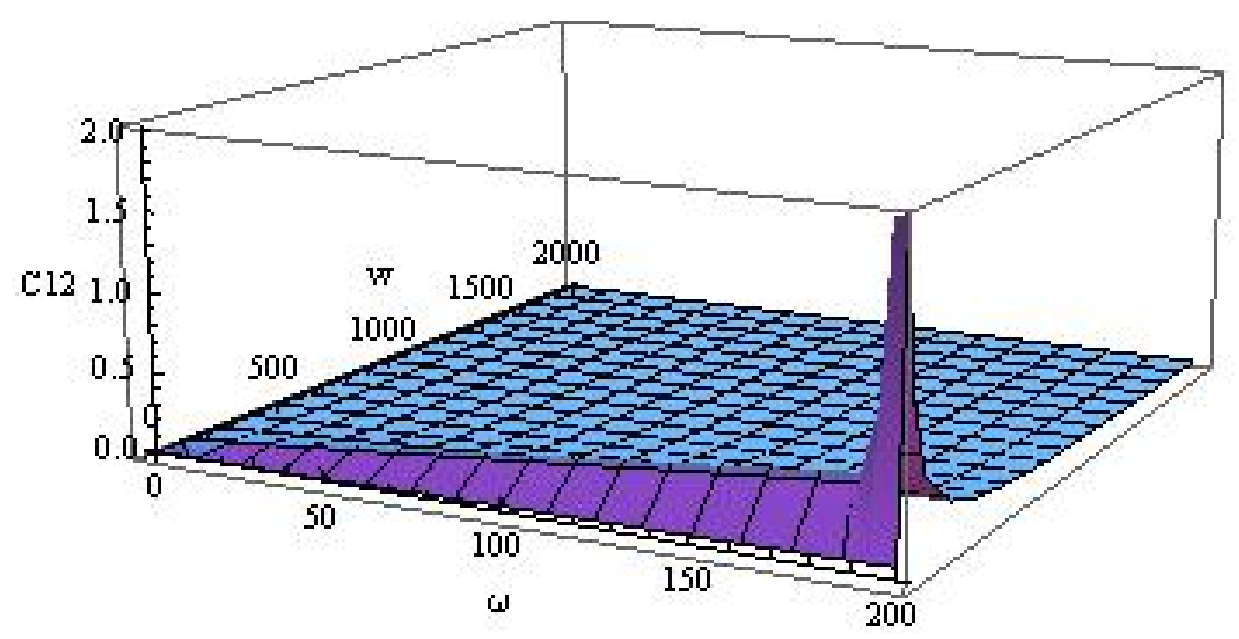

Figura 3.2-llustração de $C_{12}$ em função de $\omega$ e $w$.

Fonte: Elaborada pelo autor.

Na figura (3.2) mostramos que para que a condição adiabática seja satisfeita deve cumprir que $w>\omega$. 


\section{Superadiabaticidade}

Uma boa descrição de qualquer sistema em mecânica quântica pode ser bastante simplificada pela escolha de um quadro de referência adequado em que o correspondente Hamiltoniano seja diagonal. Assim, a descrição de um processo adiabático pode ser representada numa base instantânea dependente do tempo que diagonaliza o Hamiltoniano do sistema, esta base é chamada de base adiabática $(5,37)$. O Hamiltoniano efetivo obtido desta forma é geralmente constituído pela soma de duas contribuições: um Hamiltoniano que é diagonal instantaneamente e o acoplamento adiabático. Este último é não diagonal e contém elementos que podem impedir a evolução adiabática. O termo de acoplamento adiabático pode ser desprezado quando o Hamiltoniano muda lentamente e, se isto acontece, o processo é considerado adiabático. Entretanto isto nunca acontece na prática,pois no momento da análise e concepção de processos adiabáticos as correções não adiabáticas devem ser consideradas. Em uma série de artigos $(5,6,38,39)$, Berry mostrou que o Hamiltoniano no quadro adiabático pode ser re-transformado iterativamente em outros quadros chamados quadros superadiabáticos. Somente no quadro superadiabático pode ser avaliada com verdadeira eficácia a aproximação adiabática, já que neste quadro se conhecem os termos não adiabáticos que causam da perda de controle da dinâmica do sistema durante a evolução. Cada iteração superadiabática pode ser utilizada também para gerar um termo chamado contradiabático (40-44), que quando se adiciona ao Hamiltoniano inicial faz com que a dinâmica aproximada seja exata. Além disso pode-se gerar atalhos para a adiabaticidade, ou seja, se pode obter um Hamiltoniano que em um tempo finito mantenha as mesmas populações finais que a dinâmica adiabática inicial.

\subsection{Quadros de rotação em função do tempo}

Vamos considerar um sistema governado por um Hamiltoniano $H_{0}(t)$ dependente do tempo com níveis de energia discretos e não degenerados $E_{n}(t)$. Escolhemos uma base independente 
do tempo $\{|n\rangle\}$, e então podemos representar $H_{0}(t)$ da forma seguinte (40-42)

$$
H_{0}(t)=\sum_{n, m}|n\rangle\left\langle n\left|H_{0}(t)\right| m\right\rangle\langle m|
$$

que pode ser escrito na forma diagonal a partir do seguinte operador unitário

$$
U(t)=\sum_{n}\left|n_{0}(t)\right\rangle\langle n|
$$

sendo $\left|n_{0}(t)\right\rangle$ autoestado instantâneo do Hamiltoniano $H_{0}(t)$, ou seja,

$$
H_{0}(t)\left|n_{0}(t)\right\rangle=E_{n}\left|n_{0}(t)\right\rangle
$$

então

$$
U^{\dagger}(t) H_{0}(t) U(t)=\sum_{n} E_{n}|n\rangle\langle n| .
$$

Seja $|\psi(t)\rangle$ o vetor de estado que satisfaz a equação de Schrödinger com o Hamiltoniano $H_{0}(t)$ e $\hbar=1$, pode-se fazer a seguinte transformação $\left|\psi_{1}(t)\right\rangle=U^{\dagger}(t)|\psi(t)\rangle$, com isso pode-se obter uma equação para $\left|\psi_{1}(t)\right\rangle$, vejamos

$$
\begin{gathered}
H_{0}(t)|\psi(t)\rangle=i \frac{\partial}{\partial t}|\psi(t)\rangle \\
H_{0}(t)|\psi(t)\rangle=i\left[U(t)\left|\dot{\psi}_{1}(t)\right\rangle-U(t) \dot{U}^{\dagger}(t)|\psi(t)\rangle\right] .
\end{gathered}
$$

Logo, obtém-se a seguinte equação

$$
\left[U^{\dagger}(t) H_{0}(t) U(t)+i\left(\dot{U}^{\dagger}(t) U(t)\right)\right]\left|\psi_{1}(t)\right\rangle=i \frac{\partial}{\partial t}\left|\psi_{1}(t)\right\rangle
$$

onde podemos considerar que

$$
\begin{aligned}
& H_{0 D}(t)=U^{\dagger}(t) H_{0}(t) U(t) \\
& H_{0 A}(t)=i \dot{U}^{\dagger}(t) U(t)
\end{aligned}
$$

então, se dividimos $H_{0 A}$ em dois termos, um termo diagonal $H_{0 A D}$ e outro não diagonal $H_{0 A N D}$, a equação (4.1.4) fica assim

$$
\left[H_{0 D}+H_{0 A D}+H_{0 A N D}\right]\left|\psi_{1}(t)\right\rangle=i\left|\dot{\psi}_{1}(t)\right\rangle
$$

sendo

$$
H_{0 A D}=i \sum_{n}\left\langle\dot{n}_{0}(t) \mid n_{0}(t)\right\rangle|n\rangle\langle n|
$$




$$
H_{0 A N D}=i \sum_{n \neq m}\left\langle\dot{n}_{0}(t) \mid m_{0}(t)\right\rangle|n\rangle\langle m|
$$

Podemos observar que $H_{0 A D}$ inclui a contribuição que leva à encontrar a fase geométrica (43), enquanto o termo $H_{0 A N D}$ tem as contribuições não adiabáticas, ou seja este termo é o responsável pelo acoplamento entre os estados de $H_{0}(t)$. Além disso se pode observar que se tem dois Hamiltonianos um diagonal, $D_{0}=H_{0 D}+H_{0 A D}$ e outro não diagonal, $C_{0}=H_{0 A N D}$.

O Hamiltoniano $H_{0}(t)$ comporta-se adiabaticamente, seguindo os autovetores de $H_{0}(t)$, quando o termo $C_{0}$ é desprezível comparado com $D_{0}$. A qualidade desta aproximação adiabática é convenientemente expressa pelo fator chamado fator de adiabaticidade (37) que é dado por

$$
Q_{0}=\min _{[0, T]} \frac{\left\|D_{0}\right\|}{\left\|C_{0}\right\|}
$$

Note que se $H_{0}$ evolui adiabaticamente se satisfaz o seguinte $Q_{0}^{-1}<<1$, com isso a solução que obtemos é aproximada. A solução pode se tornar exata adicionando ao Hamiltoniano $H_{0}(t)$ um termo que chamaremos contradiabático, $H_{C D}(t)$, de modo que a equação de Schrödinger para o Hamiltoniano $H_{0}(t)+H_{C D}(t)$ admite a evolução adiabática de um vetor próprio de $H_{0}(t)$ como uma solução exata. Como falado anteriormente podemos dizer que o termo contradiabático é dado por

$$
H_{C D}^{(0)}(t)=-U(t) C_{0}(t) U^{\dagger}(t)
$$

Se somamos este termo $H_{C D}^{(0)}$ ao Hamiltoniano $H_{0}$

$$
H^{(1)}=H_{0}+H_{C D}^{(0)}
$$

se elimina os efeitos não adiabáticos responsáveis pelo acoplamento entre os autoestados de $H_{0}$, vejamos como

$$
\begin{gathered}
{\left[H_{0}(t)+H_{C D}^{(0)}(t)\right]|\psi(t)\rangle=i|\dot{\psi}(t)\rangle} \\
{\left[H_{0}(t)+H_{C D}^{(0)}(t)\right] U(t)\left|\psi_{1}(t)\right\rangle=i\left[U(t)\left|\dot{\psi}_{1}\right\rangle+\dot{U}(t)\left|\psi_{1}(t)\right\rangle\right]} \\
{\left[U^{\dagger}(t) H_{0}(t) U(t)-U^{\dagger}(t) H_{C D}^{(0)} U(t)\right]\left|\psi_{1}(t)\right\rangle=i\left[\left|\dot{\psi}_{1}\right\rangle+U^{\dagger}(t) \dot{U}(t)\left|\psi_{1}(t)\right\rangle\right]} \\
{\left[H_{0 D}-C_{0}\right]\left|\psi_{1}(t)\right\rangle=i\left|\dot{\psi}_{1}\right\rangle-\left[H_{0 A D}+H_{0 A N D}\right]\left|\psi_{1}\right\rangle}
\end{gathered}
$$

Então como $C_{0}=H_{0 A N D}$, obtemos

$$
\left[D_{0}\right]\left|\psi_{1}\right\rangle=i\left|\dot{\psi}_{1}\right\rangle
$$

Com tudo isto pode-se dizer que se o termo $C_{0}$ é o suficientemente pequeno a apro- 
ximação adiabática é boa e $H_{1}$ é diagonal na base $\left\{\left|n_{0}(t)\right\rangle\right\}$, conhecida como base adiabática. Também implica na manipulação do pequeno termo contradibático, $H_{C D}^{(0)}$, que permite a obtenção do Hamiltoniano $H^{(1)}$. Este novo Hamiltoniano proporciona um atalho para a evolução adiabática, porque mantém as populações na base instantânea do Hamiltoniano $H_{0}(t)$ invariante no instante final.

A transformação formal feita anteriormente pode ser repetida de forma iterativa para definir novos quadros. Isso se pode fazer diagonalizando o Hamiltoniano efetivo de cada quadro. Berry usou este procedimento iterativo para calcular uma seqüência de correções da fase de Berry para processos cíclicos com lentidão finita, e introduziu o conceito de superadiabaticidade (6).

A ideia da iteração superadiabatica é compreendida elaborando explicitamente o quadro seguinte. Então seja

$$
H_{1}(t)=U^{\dagger}(t) H_{0}(t) U(t)+i \dot{U}^{\dagger}(t) U(t)
$$

e a equação de Schrödinger para este Hamiltoniano é

$$
i\left|\dot{\psi}_{1}(t)\right\rangle=H_{1}\left|\psi_{1}(t)\right\rangle
$$

$H_{1}(t)$ fornece os autoestados instantâneos $\left\{\left|n_{1}(t)\right\rangle\right\}$, tal que

$$
H_{1}(t)\left|n_{1}(t)\right\rangle=E_{n}^{1}\left|n_{1}(t)\right\rangle \text {. }
$$

Agora, seja $U_{1}(t)=\sum_{n}\left|n_{1}(t)\right\rangle\langle n|$, sendo $\{|n\rangle\}$ uma base independente do tempo, com isso definimos a seguinte transformação $\left|\psi_{2}(t)\right\rangle=U_{1}^{\dagger}\left|\psi_{1}(t)\right\rangle$ e de manera análoga a anterior obtemos a seguinte equação

$$
i\left|\dot{\psi}_{2}(t)\right\rangle=H_{2}\left|\psi_{2}(t)\right\rangle
$$

sendo

$$
H_{2}(t)=U_{1}^{\dagger}(t) H_{1}(t) U_{1}(t)+i \dot{U}_{1}^{\dagger}(t) U_{1}(t)
$$

Separando o termo $i \dot{U}_{1}^{\dagger}(t) U_{1}(t)$ em duas partes como feito anteriormente, uma parte diagonal e outra não diagonal, temos

$$
\begin{aligned}
H_{1 A D} & =i \sum_{n}\left\langle\dot{n}_{1}(t) \mid n_{1}(t)\right\rangle|n\rangle\langle n| \\
H_{1 A N D} & =i \sum_{n \neq m}\left\langle\dot{n}_{1}(t) \mid m_{1}(t)\right\rangle|n\rangle\langle m| .
\end{aligned}
$$

Assim, se pode definir $D_{1}=H_{1 D}+H_{1 A D}$ e $C_{1}=H_{1 A N D}$, sendo $H_{1 D}=U_{1}^{\dagger}(t) H_{1}(t) U_{1}(t)$. Se $C_{1}$ é suficientemente pequeno a primeira ordem da aproximação adiabática na base $\left|n_{1}(t)\right\rangle$ (base superadiabática) é válida, então a dinâmica será desacoplada no novo quadro, ou seja, 
$H_{2}(t)$ é diagonal e além disso, a solução será aproximada. Agora se quisermos que a solução seja exata, devemos somar o termo contradiabático, $H_{C D}^{(1)}$, ao Hamiltoniano $H_{0}$, então obtemos um novo Hamiltoniano

$$
H^{(2)}(t)=H_{0}(t)+H_{C D}^{(1)}(t)
$$

sendo

$$
H_{C D}^{(1)}(t)=-U(t) U_{1}(t) C_{1}(t) U_{1}^{\dagger}(t) U^{\dagger}(t)
$$

Pode acontecer que um processo não seja adiabático, se $C_{0}$ não puder ser desprezado, mas na primeira ordem na base superadiabática, $\left\{\left|n_{1}(t)\right\rangle\right\}, C_{1}$ poderia ser desprezado. Ou seja a qualidade desta aproximação adiabática é dada por

$$
Q_{1}=\min _{[0, T]} \frac{\left\|D_{1}\right\|}{\left\|C_{1}\right\|}
$$

Para mais iterações definimos quadros adiabáticos de ordem superior, tal que a equação de Schrödinger neste quadro é

$$
i\left|\dot{\psi}_{j}(t)\right\rangle=H_{j}(t)\left|\psi_{j}(t)\right\rangle
$$

onde

$$
H_{j+1}(t)=U_{j}^{\dagger}(t) H_{j}(t) U_{j}(t)+i \dot{U}_{j}^{\dagger}(t) U_{j}(t)
$$

com

$$
\begin{gathered}
U_{j}(t)=\sum\left|n_{j}(t)\right\rangle\langle n| \\
H_{j}(t)\left|n_{j}(t)\right\rangle=E_{n}^{(j)}(t)\left|n_{j}(t)\right\rangle
\end{gathered}
$$

Separando o termo $i \dot{U}_{j}^{\dagger}(t) U_{j}(t)$ numa parte diagonal e outra não diagonal, ou seja

$$
\begin{aligned}
H_{j A D} & =i \sum_{n}\left\langle\dot{n}_{j}(t) \mid n_{j}(t)\right\rangle|n\rangle\langle n| \\
H_{j A N D} & =i \sum_{n \neq m}\left\langle\dot{n}_{j}(t) \mid m_{j}(t)\right\rangle|n\rangle\langle m| .
\end{aligned}
$$

Assim, $D_{j}=H_{j D}+H_{j A D}$ e $C_{j}=H_{j A N D}$, sendo $H_{j D}=U_{j}^{\dagger}(t) H_{j}(t) U_{j}(t)$. Além disso, o fator de adiabaticidade está dada por

$$
Q_{j}=\min _{[0, T]} \frac{\left\|D_{j}\right\|}{\left\|C_{j}\right\|} .
$$

Se $Q_{j}$ é suficientemente grande, então no j-ésimo quadro, podemos fazer a aproximação adiabática, ou seja, desprezar o termo $C_{j}$. Agora se quisermos que a solução seja exata e não 


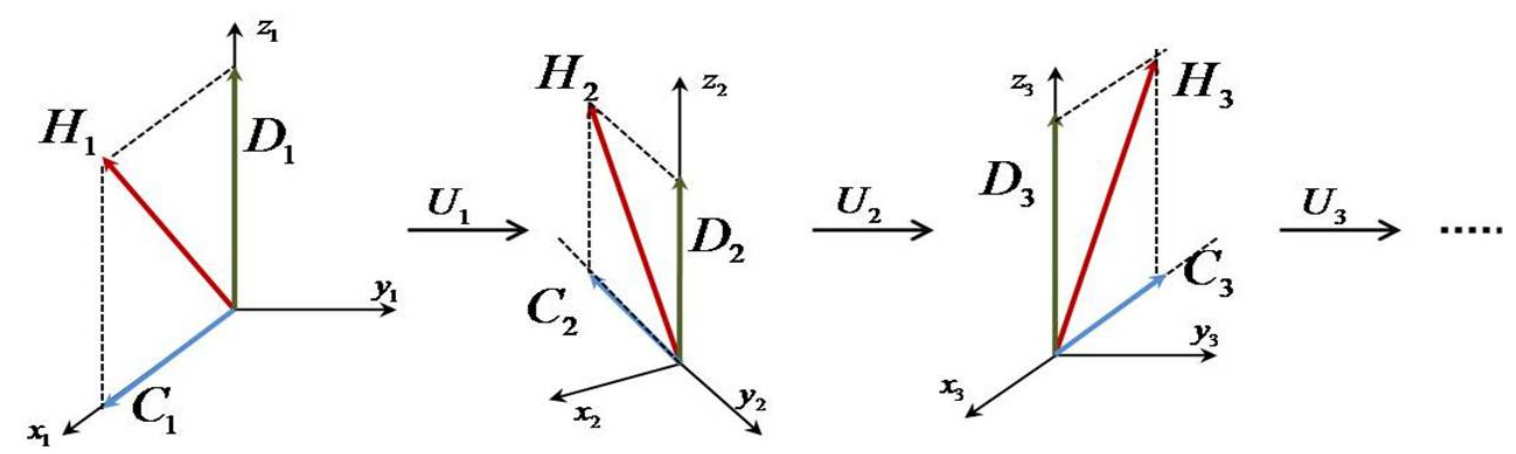

$1^{\circ}$ quadro adiabático $\quad 2^{\circ}$ quadro adiabático $\quad 3^{\circ}$ quadro adiabático

Figura 4.1-Representação gráfica dos quadros de rotação.

Fonte: Elaborada pelo autor.

aproximada, teremos que somar o termo contradiabatico, $H_{C D}^{(j)}$, ao Hamiltoniano $H_{0}$, obtendo

$$
H^{j+1}=H_{0}+H_{C D}^{j}
$$

com

$$
H_{C D}^{(j)}(t)=-U(t) U_{1} \ldots U_{j}(t) C_{j}(t) U_{j}^{\dagger}(t) \ldots U_{1}^{\dagger}(t) U^{\dagger}(t)
$$

\subsection{Superadibaticidade para sistemas abertos}

Agora vamos generalizar o que foi discutido na seção anterior para o caso de evoluções não unitárias $(11,43)$. Vamos considerar a equação mestra na forma de Lindblad (3.5.6)

$$
\mathcal{L}(t)|\rho(t)\rangle\rangle=|\dot{\rho}(t)\rangle\rangle
$$

Lembrando que para utilizar o formalismo introduzido no capitulo (3) é preciso escrever o superoperador como uma matriz e a matriz densidade como vetor $(11,45)$. Começamos definindo como $B$ o conjunto de bases independentes do tempos no espaço de dimensão $D^{2}$ (onde $\mathrm{D}$ é a dimensão do espaço de Hilbert). Um exemplo de base desse tipo e a composta pelas três matrizes de Pauli e a matriz identidade, para o caso da partícula com spin 1/2.

A supermatriz $\mathcal{L}(t)$ pode não ser Hermitiana. Nesse caso, $\mathcal{L}(t)$ pode ser não-diagonalizável, mas é sempre possível encontrar uma transformação de similaridade $S(t)$, de tal modo que $\mathcal{L}(t)$ é escrito na forma canônica de Jordan

$$
\mathcal{L}_{J}(t)=S^{\dagger}(t) \mathcal{L}(t) S(t)=\operatorname{diag}\left[J_{1}(t), \ldots, J_{m}\right]
$$


onde $J_{i}$ representa o bloco de Jordan (de dimensão $n_{\alpha}$ ) correspondente ao autovalor $\lambda_{\alpha}$ de $\mathcal{L}(t)$. O número $m$ de blocos de Jordan é igual ao número de autovetores linearmente independentes de $\mathcal{L}(t)$. A transformação de similaridade é dada por

$$
\left.S(t)=\sum_{\alpha=1}^{m} \sum_{j=0}^{n_{\alpha}-1}\left|D_{\alpha}^{(j)}\right\rangle\right\rangle\left\langle\left\langle\sigma_{\alpha}^{(j)}\right|\right.
$$

onde $\left.\left\{\left|D^{(j)_{\alpha}}\right\rangle\right\rangle\right\}$ é a base formada pelos autovetores instantâneos direitos de $\mathcal{L}(t)$ associados ao conjunto de autovalores $\left\{\lambda_{\alpha}\right\}(11)$, e $\left\{\left\langle\left\langle\sigma_{\alpha}^{(j)}\right|\right\}\right.$ são os vetores da base $B$ introduzida anteriormente. A transformação inversa $S^{-1}(t)$, tal que $S^{-1}(t) S(t)=S(t) S^{-1}(t)=\mathbb{I}$, pode ser definida pelos autovetores instantâneos esquerdos de $\mathcal{L}(t)$. Explorando a equivalência formal entre a equação (3.5.6) e a equação de Schrödinger com um Hamiltoniano não Hermitiano, os argumentos dos quadros de rotação em função do tempo ilustrados na seção anterior podem ser usados aqui. Aplicando a transformação de $S^{-1}(t)$ para ambos lados da equação (3.5.6), obtemos depois de uma manipulação o seguinte

$$
\left.\left.\left[\mathcal{L}_{J}(t)+\mathcal{L}_{D}(t)+\mathcal{L}_{N D}(t)\right]|\rho\rangle\right\rangle_{J}=|\dot{\rho}\rangle\right\rangle_{J}
$$

onde

$$
\begin{aligned}
\mathcal{L}_{D}(t) & \left.=\sum\left|\sigma_{\alpha}^{(j)}\right\rangle\right\rangle C_{\alpha, \alpha}^{j, j}(t)\left\langle\left\langle\sigma_{\alpha}^{(j)}\right|\right. \\
\mathcal{L}_{N D}(t) & \left.=\sum_{\alpha \neq \alpha^{\prime}}\left|\sigma_{\alpha}^{(j)}\right\rangle\right\rangle C_{\alpha, \alpha^{\prime}}^{j, j^{\prime}}(t)\left\langle\left\langle\sigma_{\alpha^{\prime}}^{\left(j^{\prime}\right)}\right|\right.
\end{aligned}
$$

$\operatorname{com} C_{\alpha, \alpha^{\prime}}^{j, j^{\prime}}(t)=\left\langle\left\langle\sigma_{\alpha}^{(j)}\left|\dot{S}^{-1}(t) S(t)\right| \sigma_{\alpha^{\prime}}^{\left(j^{\prime}\right)}\right\rangle\right\rangle$. O índice $J$ indica que a matriz $\mathcal{L}(t)$ está na forma de Jordan e o vetor de coerência é transformado assim $\left.|\rho\rangle\rangle_{J}=S^{-1}|\rho\rangle\right\rangle$.

Para sistemas abertos, o problema da condução sem transição quântica consiste em encontrar o termo contradibático $\mathcal{L}_{C D}(t)$, de tal forma que os diferentes blocos de Jordan de $\mathcal{L}$ evoluam de forma independente sob a ação de $\mathcal{L}(t)+\mathcal{L}_{C D}(t)$. Uma vez que os termos $\mathcal{L}_{J}(t)$ e $\mathcal{L}_{D}(t)$ preservam a estrutura dos blocos de Jordan, qualquer mistura entre os diferentes blocos de Jordan é obrigado a surgir a partir de $\mathcal{L}_{N D}(t)$. Portanto, através da utilização da mesma abordagem feita na seção anterior, pode-se inferir o termo contradiabático $\mathcal{L}_{C D}(t)$ como

$$
\mathcal{L}_{C D}(t)=-S(t) \mathcal{L}_{N D}(t) S^{-1}(t)
$$

Tal como no caso do sistema fechado, $\mathcal{L}_{C D}(t)$ é o termo que deve ser somado de modo que o estado do sistema, em toda a evolução, não mude. Além disso a supermatriz $\mathcal{L}(t)$ pode ser re-transformado iterativamente em quadros superadiabaticos onde a aproximação adiabática pode ser avaliada com eficácia, também pode-se construir o termo contradiabático nesta base 
superadiabática. No próximo capítulo veremos exemplos de aplicação de toda a teoria exposta até aqui em sistemas de RMN. 


\section{Aplicações em RMN}

Neste capítulo faremos três aplicações para estudar a condição adiabática para sistemas fechados e abertos em RMN.

\subsection{Primeira Aplicação}

Vamos considerar uma partícula com spin $1 / 2$ na presença de um campo magnético do tipo $\vec{B}(t)=B_{0} \hat{z}+B_{1}(\cos \omega t \hat{\imath}+\operatorname{sen} \omega t \hat{\jmath})$ como na referencia (46) então o Hamiltoniano que representa o acoplamento spin-campo magnético é

$$
H(t)=\frac{\omega_{0}}{2} \sigma_{z}+\frac{\omega_{1}}{2}\left(\sigma_{x} \cos \omega t+\sigma_{y} \operatorname{sen} \omega t\right)
$$

ou

$$
H(t)=\frac{\omega_{0}}{2} \sec \theta\left(\begin{array}{cc}
\cos \theta & e^{-i \omega t} \operatorname{sen} \theta \\
e^{i \omega t} \operatorname{sen} \theta & \cos \theta
\end{array}\right)
$$

sendo $\omega_{0}$ a frequência de Larmor, $\omega$ é a frequência de rotação do pulso de RF e $R=\tan \theta=$ $\omega_{1} / \omega_{0}$. Os autovetores e autovalores são

$$
\begin{gathered}
\left|\varphi_{+}(t)\right\rangle=\left(\begin{array}{c}
\cos \frac{\theta}{2} \\
e^{i \omega t} \operatorname{sen} \frac{\theta}{2}
\end{array}\right) \quad\left|\varphi_{-}(t)\right\rangle=\left(\begin{array}{c}
-e^{-i \omega t} \operatorname{sen} \frac{\theta}{2} \\
\cos \frac{\theta}{2}
\end{array}\right) \\
E_{ \pm}= \pm \sec \theta \frac{\omega_{0}}{2} .
\end{gathered}
$$

Resolvendo a equação de Schrödinger considerando que o estado estado inicial é $\left|\varphi_{+}(0)\right\rangle=$ $\left(\begin{array}{c}\cos \frac{\theta}{2} \\ \operatorname{sen} \frac{\theta}{2}\end{array}\right)$, obtemos como solução

$|\psi(t)\rangle=\left[\cos \frac{\varpi t}{2}-i\left(\frac{\omega_{0} \sec \theta-\omega \cos \theta}{\varpi}\right) \operatorname{sen} \frac{\varpi t}{2}\right] e^{-i \omega t / 2}\left|\varphi_{+}(t)\right\rangle-i\left[\frac{\omega \operatorname{sen} \theta}{\varpi} \operatorname{sen} \frac{\varpi t}{2}\right] e^{i \omega t / 2}\left|\varphi_{-}(t)\right\rangle$

sendo $\varpi=\omega_{0} \sqrt{(1-k)^{2}+R^{2}}$ e $k=\omega / \omega_{0}$ 


$$
\begin{gathered}
\text { Seja o operador unitario } U(t)=\left(\begin{array}{cc}
\cos \frac{\theta}{2} & -e^{-i \omega t} \operatorname{sen} \frac{\theta}{2} \\
e^{i \omega t} \operatorname{sen} \frac{\theta}{2} & \cos \frac{\theta}{2}
\end{array}\right) \text {, então } \\
H_{0 A}(t)=i \dot{U}^{\dagger}(t) U(t)=\left(\begin{array}{cc}
\omega \operatorname{sen}^{2} \frac{\theta}{2} & \frac{\omega}{2} e^{-i \omega t} \operatorname{sen} \theta \\
\frac{\omega}{2} e^{i \omega t} \operatorname{sen} \theta & -\omega \operatorname{sen}^{2} \frac{\theta}{2}
\end{array}\right)
\end{gathered}
$$

então os termo que estão fora da diagonal são as contribuições não adiabáticas, ou seja, esses termos são os responsáveis pelo acoplamento entre os estados de $H(t)$

$$
H_{0 N D}(t)=\left(\begin{array}{cc}
0 & \frac{\omega}{2}-e^{-i \omega t} \operatorname{sen} \theta \\
\frac{\omega}{2} e^{i \omega t} \operatorname{sen} \theta & 0
\end{array}\right) \text {. }
$$

Se definimos $F(t)=\left|\left\langle\psi(t) \mid \varphi_{n}(t)\right\rangle\right|$, que representa o valor do grau de similaridade que o elemento representado tem com o elemento tomado como base na comparação, então se $F(t)$ é igual a 1 a condição adiabática (3.3.8) é satisfeita

$$
\begin{gathered}
\left|\frac{\left\langle\varphi_{n}(t) \mid \dot{\varphi}_{m}(t)\right\rangle}{E_{m}-E_{n}}\right| \ll 1 \\
\left(Q_{0}\right)^{-1}=\frac{k \operatorname{sen} \theta}{2 \sec \theta} \ll 1
\end{gathered}
$$

para nosso exemplo $F(t)$ é

$$
F(t)=\left[1-\frac{(k \operatorname{sen} \theta)^{2}}{(1-k)^{2}+R^{2}} \operatorname{sen}^{2} \frac{\varpi t}{2}\right]^{1 / 2} .
$$

Seja $\omega_{0}=1700 \mathrm{~Hz}$ e $\omega_{1}=100 \mathrm{~Hz}$, então vamos analisar dois casos o primeiro com $\omega=\omega_{0}$ e o segundo com $\omega=10 \omega_{0}$, com essas informações obtemos o gráfico (5.1), que mostra como muda $F(t)$ nos dois casos.

Substituindo os dados na equação (5.1.6) para os dois casos, se verifica que para o primeiro caso onde $\omega=\omega_{0}$ a condição adiabática é satisfeita, $\left(Q_{0}\right)^{-1}=0.058 \ll 1$, enquanto para o segundo caso $\omega=10 \omega_{0}$ a condição adiabática não é satisfeita, $\left(Q_{0}\right)^{-1}=0.58$. Pode se observar que para $\omega=\omega_{0}$ se tem que $F(t) \neq 1$, ou seja, que o estado final não pode se aproximar ao seguinte estado $e^{i \phi}\left|\varphi_{n}\right\rangle$, enquanto para $\left(\omega=10 \omega_{0}\right)$ se tem que $F(t) \approx 1$ mas a condição adiabática não é satisfeita (5.1.6), então podemos observar alguns conflitos que poderíamos resolver levando ao Hamiltoniano a uma nova base, base superadiabática, ou somando o termo contradiabático.

Acharemos primeiro o termo contradiabático

$$
H_{C D}^{(0)}(t)=-U(t) H_{0 N D}(t) U^{\dagger}(t)
$$




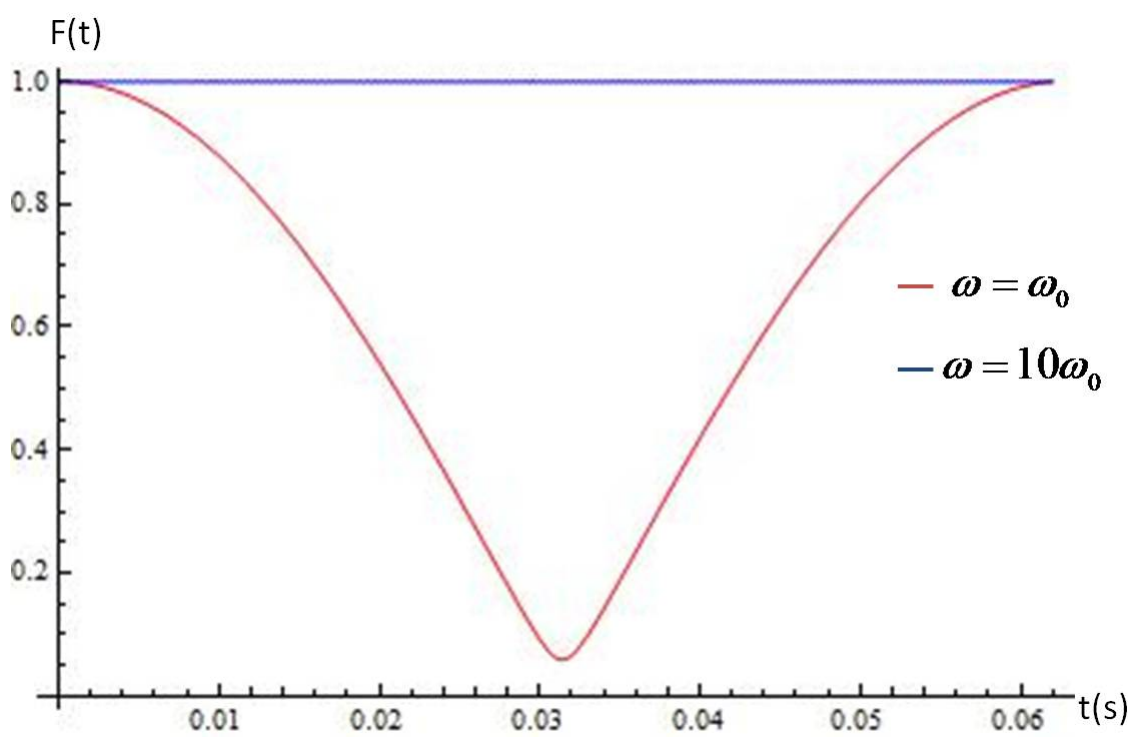

Figura 5.1-Gráfico de $F(t)$ comparando os dois casos, $\omega=\omega_{0}$ e $\omega=10 \omega_{0}$.

Fonte: Elaborada pelo autor.

$$
H_{C D}^{(0)}(t)=\left(\begin{array}{cc}
\frac{\omega}{2} \operatorname{sen}^{2} \theta & -\frac{\omega}{2} e^{-i \omega t} \operatorname{sen} \theta \\
-\frac{\omega}{4} e^{i \omega t} \operatorname{sen} \theta & -\frac{\omega}{2} \operatorname{sen}^{2} \theta
\end{array}\right)
$$

Agora se somamos $H_{C D}(t)$ ao Hamiltoniano inicial $H(t)$, então a solução da equação de Schrödinger do Hamiltoniano $H^{(1)}(t)=H(t)+H_{C D}^{(0)}(t)$ é

$$
\left|\psi_{0}^{(1)}(t)\right\rangle=e^{-i \frac{\omega_{0} t}{2} \sec \theta} e^{-i \frac{\omega t}{2}(1-\cos \theta)}\left|\varphi_{+}(t)\right\rangle
$$

sendo $-\frac{\omega_{0} t}{2} \sec \theta$ a fase dinâmica e $-\frac{\omega t}{2}(1-\cos \theta)$ a fase geométrica.

\section{- iteração superadiabática}

Levaremos ao Hamiltoniano $H(t)$ a um novo quadro para ver se neste novo quadro se pode fazer a aproximação adiabática e também encontrar o termo contradiabático.

$$
\begin{gathered}
H_{1}(t)=U^{\dagger}(t) H(t) U(t)+i \dot{U}^{\dagger}(t) U(t) \\
H_{1}(t)=\frac{\omega_{0}}{2}\left(\begin{array}{cc}
\sec \theta+2 k \operatorname{sen}^{2} \frac{\theta}{2} & k \operatorname{sen} \theta e^{-i \omega t} \\
k \operatorname{sen} \theta e^{i \omega t} & -\left(\sec \theta+2 k \operatorname{sen}^{2} \frac{\theta}{2}\right)
\end{array}\right)
\end{gathered}
$$

Fazendo uma mudança de variáveis

$$
\begin{gathered}
r_{1} \cos \theta_{1}=\sec \theta+2 k \operatorname{sen}^{2} \frac{\theta}{2} \\
r_{1} \operatorname{sen} \theta_{1}=k \operatorname{sen} \theta
\end{gathered}
$$




$$
r_{1}=\sqrt{(k \operatorname{sen} \theta)^{2}+\left(\sec \theta+2 k \operatorname{sen}^{2} \frac{\theta}{2}\right)^{2}}
$$

então o Hamiltoniano $H_{1}(t)$ fica

$$
H_{1}(t)=\frac{\omega_{0} r_{1}}{2}\left(\begin{array}{cc}
\cos \theta_{1} & e^{-i \omega t} \operatorname{sen} \theta_{1} \\
e^{i \omega t} \operatorname{sen} \theta & -\cos \theta_{1}
\end{array}\right),
$$

os autovetores e autovalores são

$$
\begin{gathered}
\left|\varphi_{+}^{1}(t)\right\rangle=\left(\begin{array}{c}
\cos \frac{\theta_{1}}{2} \\
e^{i \omega t} \operatorname{sen} \frac{\theta_{1}}{2}
\end{array}\right) ; \quad E_{+}^{1}=\frac{\omega_{0}}{2} r_{1} \\
\left|\varphi_{-}^{1}(t)\right\rangle=\left(\begin{array}{c}
-e^{-i \omega t} \operatorname{sen} \frac{\theta_{1}}{2} \\
\cos \frac{\theta_{1}}{2}
\end{array}\right) ; \quad E_{-}^{1}=-\frac{\omega_{0}}{2} r_{1} .
\end{gathered}
$$

Agora podemos construir o operador unitário $U_{1}(t)$

$$
U_{1}(t)=\left(\begin{array}{cc}
\cos \frac{\theta_{1}}{2} & -e^{-i \omega t} \operatorname{sen} \frac{\theta_{1}}{2} \\
e^{i \omega t} \operatorname{sen} \frac{\theta_{1}}{2} & \cos \frac{\theta_{1}}{2}
\end{array}\right),
$$

com o qual faremos a transformação do Hamiltoniano $H_{1}(t)$, logo

$$
H_{2}(t)=U_{1}^{\dagger}(t) H_{1}(t) U_{1}(t)+i \dot{U}_{1}^{\dagger}(t) U_{1}(t)
$$

$\log 0$

$$
H_{1 A}(t)=i \dot{U}_{1}^{\dagger}(t) U_{1}(t)=\left(\begin{array}{cc}
\omega \operatorname{sen}^{2} \frac{\theta_{1}}{2} & -\frac{\omega}{2} e^{-i \omega t} \operatorname{sen} \theta_{1} \\
\frac{\omega}{2} e^{i \omega t} \operatorname{sen} \theta_{1} & -\omega \operatorname{sen}^{2} \frac{\theta_{1}}{2}
\end{array}\right)
$$

com os elementos que estão fora da diagonal podemos obter a matriz $H_{1 A N D}(t)$

$$
H_{1 N D}(t)=\left(\begin{array}{cc}
0 & -\frac{\omega}{2} e^{-i \omega t} \operatorname{sen} \theta_{1} \\
\frac{\omega}{2} e^{i \omega t} \operatorname{sen} \theta_{1} & 0
\end{array}\right)
$$

e com isso a condição adiabática é

$$
\left(Q_{1}\right)^{-1}=\frac{\omega \operatorname{sen} \theta_{1}}{2 \omega_{0} r_{1}} \ll 1 .
$$

Para o caso $\omega_{0}=\omega$ pode-se mostrar que a condição adiabática (5.1.17) é satisfeita, $\left(Q_{1}\right)^{-1}=$ 0.029, então fazendo a aproximação adiabática a solução da equação de Schrödinger do Hamiltoniano $H_{2}(t)$ é

$$
\left|\psi_{2}(t)\right\rangle=\exp \left(-i \frac{\omega_{0}}{2} r_{1}-i \omega \operatorname{sen}^{2} \frac{\theta_{1}}{2}\right)\left|\varphi_{+}^{1}(0)\right\rangle
$$


no sistema do laboratorio

$$
\left|\psi_{0}^{(2)}(t)\right\rangle=U(t) U_{1}(t)\left|\psi_{2}(t)\right\rangle
$$

ou

$$
\left|\psi_{0}^{(2)}(t)\right\rangle=\left(\begin{array}{c}
\cos \left(\frac{\theta+\theta_{1}}{2}\right) \\
e^{i \omega t} \operatorname{sen}\left(\frac{\theta+\theta_{1}}{2}\right)
\end{array}\right) \exp \left(-i \frac{\omega_{0}}{2} r_{1}-i \omega \operatorname{sen}^{2} \frac{\theta_{1}}{2}\right)
$$

com isso podemos obter o gráfico (5.2) de $F(t)=\left|\left\langle\psi_{0}^{(2)}(t) \mid \varphi_{+}(t)\right\rangle\right|$, para poder observar se ainda há inconsistência.

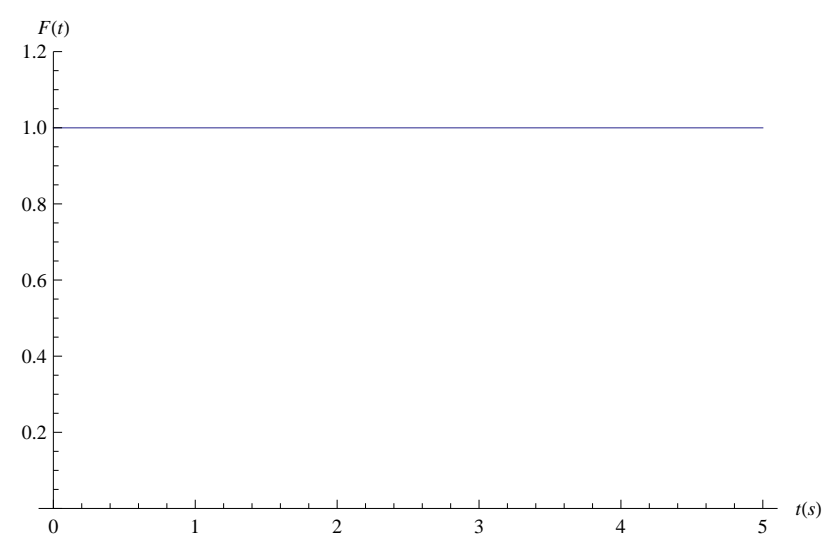

Figura 5.2-Gráfica de $F(t)$ para $\omega=\omega_{0}$ no segundo quadro.

Fonte: Elaborada pelo autor.

Como se pode observar que a inconsistência que apresentava no inicio sumiu, ou seja, o estado final pode se aproximar $e^{\phi}\left|\varphi_{n}(t)\right\rangle$, então é melhor fazer a aproximação adiabática neste quadro ou também construir o termo contradiabático.

\subsection{Segunda Aplicação}

Vamos considerar o mesmo Hamiltoniano do exemplo 1, só que agora o pulso ainda de radiofrequência, mas com amplitude modulada. Então

$$
H(t)=\frac{\omega_{0}}{2} \sigma_{z}+\frac{\omega_{1}(t)}{2}\left(\sigma_{x} \cos \phi(t)+\sigma_{y} \operatorname{sen} \phi(t)\right)
$$

para simplificar as contas vamos fazer uma rotação de $\phi(t)$ em torno do $\sigma_{z}$, logo o Hamiltoniano fica assim

$$
H_{0}(t)=\frac{\left(\omega_{0}-\dot{\phi}(t)\right)}{2} \sigma_{z}+\frac{\omega_{1}(t)}{2} \sigma_{x}
$$


os autovetores e autovalores são

$$
\begin{gathered}
\left|\varphi_{+}(t)\right\rangle=\left(\begin{array}{c}
\cos \frac{\theta}{2} \\
\operatorname{sen} \frac{\theta}{2}
\end{array}\right) \quad E_{+}(t)=\frac{1}{2} \sqrt{\omega_{1}^{2}+(\omega-\dot{\phi})^{2}} \\
\left|\varphi_{-}(t)\right\rangle=\left(\begin{array}{c}
-\operatorname{sen} \frac{\theta}{2} \\
\cos \frac{\theta}{2}
\end{array}\right) \quad E_{-}(t)=-\frac{1}{2} \sqrt{\omega_{1}^{2}+(\omega-\dot{\phi})^{2}}
\end{gathered}
$$

sendo $\tan \theta=\frac{\omega_{1}}{\omega-\dot{\phi}} \log$ nosso operador unitário é

$$
U_{0}(t)=\left(\begin{array}{cc}
\cos \frac{\theta}{2} & -\operatorname{sen} \frac{\theta}{2} \\
\operatorname{sen} \frac{\theta}{2} & \cos \frac{\theta}{2}
\end{array}\right) .
$$

Então fazendo a primeira iteração obtemos

$$
H_{1}(t)=U^{\dagger}(t) H_{0}(t) U(t)+i \dot{U}^{\dagger}(t) U(t)
$$

onde se pode mostrar que

$$
H_{0 A}(t)=i \dot{U}^{\dagger}(t) U(t)=i \frac{\dot{\theta}}{2}\left(\begin{array}{cc}
0 & 1 \\
-1 & 0
\end{array}\right)
$$

e

$$
H_{0 A N D}(t)=H_{0 A}(t)=-\frac{\dot{\theta}}{2} \sigma_{y}
$$

a condição adiabática é

$$
\left(Q_{0}\right)^{-1}=\left|\frac{\dot{\theta}}{\sqrt{\omega_{1}^{2}+\left(\omega_{0}-\dot{\phi}\right)^{2}}}\right| \ll 1
$$

e o termo contradibático é

$$
H_{C D}^{0}=-U(t) H_{0 A N D} U^{\dagger}(t)=\frac{\dot{\theta}}{2} \sigma_{y} .
$$

Se a condição adiabática não for satisfeita podemos fazer outra transformação de bases. Então vamos obter que os autovalores e autovetores de

$$
H_{1}(t)=\left(\begin{array}{cc}
E_{+}(t) & i \frac{\dot{\theta}}{2} \\
-i \frac{\dot{\theta}}{2} & E_{-}(t)
\end{array}\right)
$$

são

$$
\begin{gathered}
\left|\varphi_{+}^{1}(t)\right\rangle=\left(\begin{array}{c}
\cos \frac{\theta_{1}}{2} \\
-i \operatorname{sen} \frac{\theta_{1}}{2}
\end{array}\right) \quad, \quad E_{+}^{1}(t)=\frac{1}{2} \sqrt{\omega_{1}^{2}+(\omega-\dot{\phi})^{2}+\dot{\theta}^{2}} \\
\left|\varphi_{-}^{1}(t)\right\rangle=\left(\begin{array}{c}
-i \operatorname{sen} \frac{\theta_{1}}{2} \\
\cos \frac{\theta_{1}}{2}
\end{array}\right) \quad, \quad E_{-}^{1}(t)=-\frac{1}{2} \sqrt{\omega_{1}^{2}+(\omega-\dot{\phi})^{2}+\dot{\theta}^{2}}
\end{gathered}
$$


sendo $\tan \theta_{1}=\frac{\dot{\theta}}{2 E_{+}} \operatorname{logo}$ nosso operador unitario é

$$
U_{1}(t)=\left(\begin{array}{cc}
\cos \frac{\theta_{1}}{2} & -i \operatorname{sen} \frac{\theta_{1}}{2} \\
-i \operatorname{sen} \frac{\theta_{1}}{2} & \cos \frac{\theta_{1}}{2}
\end{array}\right)
$$

então fazendo a segunda iteração obtemos

$$
H_{2}(t)=U_{1}^{\dagger}(t) H_{1}(t) U_{1}(t)+i \dot{U}_{1}^{\dagger}(t) U_{1}(t)
$$

se pode obter

$$
H_{1 A}(t)=i \dot{U}_{1}^{\dagger}(t) U_{1}(t)=\frac{\dot{\theta}_{1}}{2}\left(\begin{array}{cc}
0 & -1 \\
-1 & 0
\end{array}\right)
$$

e também

$$
H_{1 A N D}(t)=H_{1 A}(t)=-\frac{\dot{\theta}}{2} \sigma_{x}
$$

Então condição adiabática é

$$
\left(Q_{1}\right)^{-1}=\left|\frac{\dot{\theta}_{1}}{\sqrt{\omega_{1}^{2}+\left(\omega_{0}-\dot{\phi}\right)^{2}+\dot{\theta}^{2}}}\right| \ll 1
$$

e o termo contradibático é

$$
H_{C D}^{1}=-U(t) U_{1}(t) H_{0 A N D} U_{1}^{\dagger}(t) U^{\dagger}(t)=\frac{\dot{\theta}}{2}\left[\cos \theta \sigma_{x}-\operatorname{sen} \theta \sigma_{z}\right] .
$$

Pode se observar que a estrutura do $H_{C D}^{j}$ muda com $j$, isto é uma das razões pela qual iterações de ordem superior são atraentes, já que outras iterações definem quadros de ordem superior. De forma semelhante como se obteve as equações (5.2.4) e (5.2.7), se pode fazer outras iterações, obtendo para o n-ésimo quadro

$$
Q_{n}=\left|\frac{\sqrt{\omega_{1}^{2}+\left(\omega_{0}-\dot{\phi}\right)^{2}+\left(\dot{\theta}_{0}\right)^{2}+\left(\dot{\theta}_{1}\right)^{2}+\ldots+\left(\dot{\theta}_{n-1}\right)^{2}}}{\dot{\theta_{n}}}\right| .
$$

Vamos a considerar como exemplo numérico, que o pulso aplicado é um pulso adiabático $(7,8,47,48)$ da seguinte forma

$$
\begin{aligned}
\omega_{0}-\dot{\phi}(t)=\Delta \omega & =10^{4} \times \pi \tanh \left[5.3\left(1-\frac{2 t}{2 \times 10^{3}}\right)\right] \\
\omega_{1}(t) & =10^{4} \times \pi \operatorname{sech}\left[5.3\left(\frac{2 t}{2 \times 10^{3}}-1\right)\right] .
\end{aligned}
$$

Para um processo fixo os termos contradiabáticos, $H_{C D}^{j}$ tendem a diminuir conforme aumenta $j$, até um valor em que eles começam a crescer, tal como se mostra na figura (5.6) 


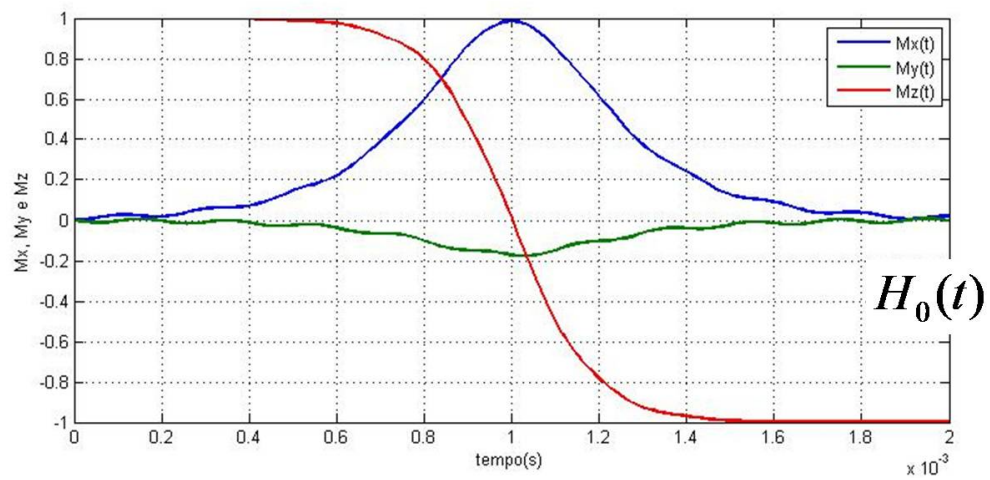

Figura 5.3-Evolução da magnetização no referencial girante.

Fonte: Elaborada pelo autor.

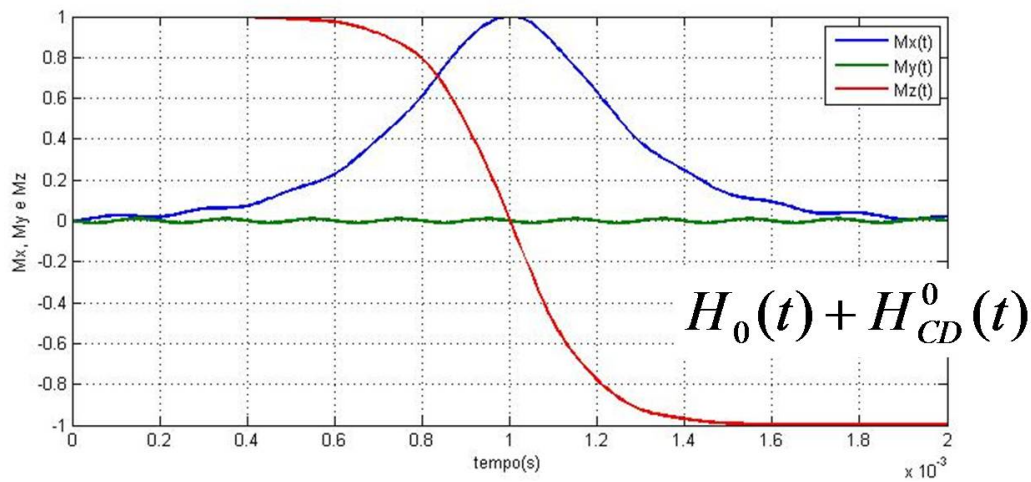

Figura 5.4 - Evolução da magnetização no referencial girante, quando se soma ao Hamiltoniano inicial o termo $H_{C D}^{0}$.

Fonte: Elaborada pelo autor.

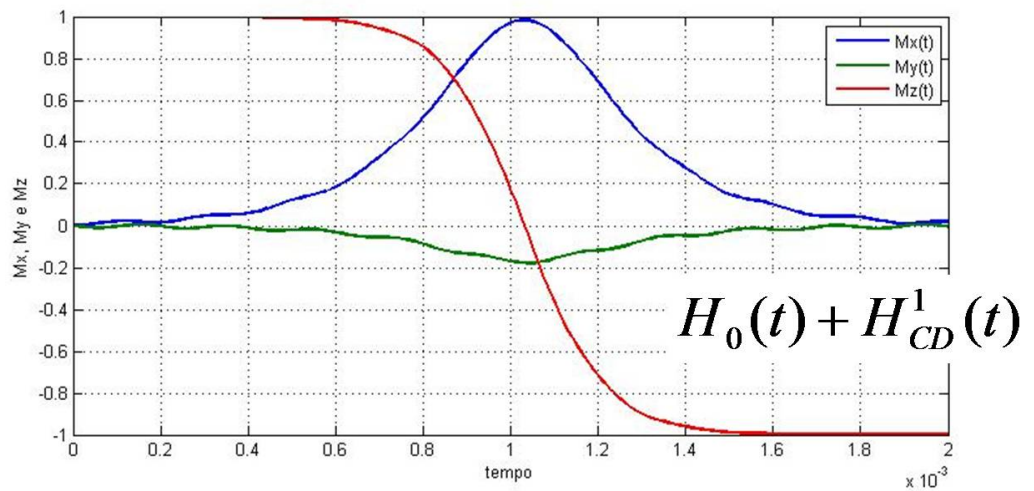

Figura 5.5 - Evolução da magnetização no referencial girante, quando se soma ao Hamiltoniano inicial o termo $H_{C D}^{1}$.

Fonte: Elaborada pelo autor. 


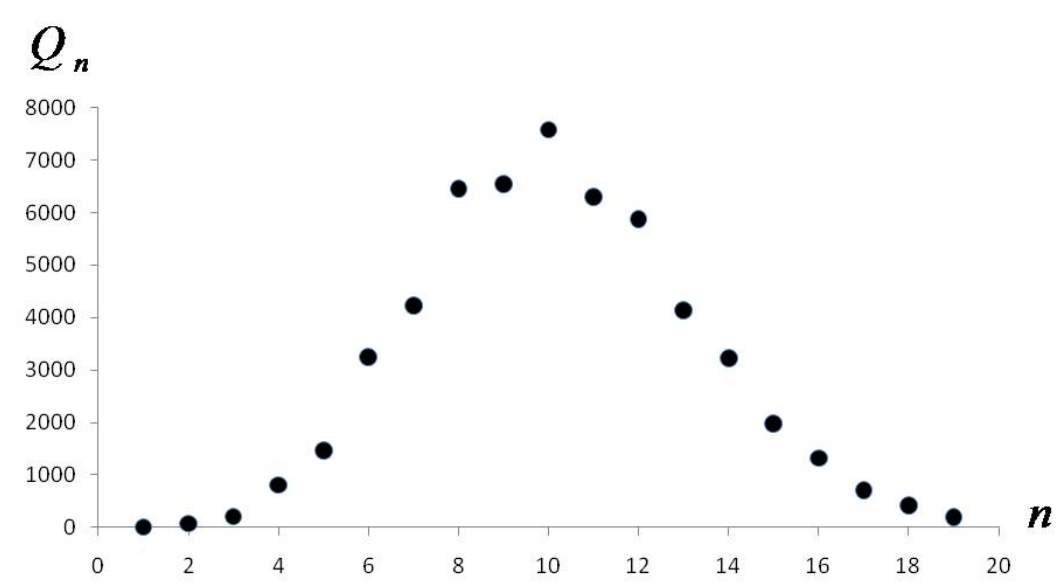

Figura 5.6-Fator da adiabaticidade $Q_{n}$ para nosso pulso sech/tanh.

Fonte: Elaborada pelo autor.

e nas referência $(37,44)$. Isto significa que em algum quadro super-adiabático se pode fazer o truncamento da iteração, isto é quando $\left(Q_{n}\right)^{-1} \ll 1$, com isso poderíamos ter um bom processo adiabático, para nosso caso podemos ver na figura (5.6) que a iteração poderia chegar até $Q_{10}=7575$, ou seja podemos truncar antes de essa iteração, já que depois o $Q_{n}$ começa a diminuir, isso quer dizer que depois o pulso começa a deixar de ser adiabático. O truncamento depende de quão adiabático queremos que seja o pulso.

Na figura (5.4) podemos ver que a magnetização se inverte no plano $x-z$ já que a componente da magnetização em $y$ desaparece, isso acontece porque ao somar o primeiro termo $H_{C D}^{0}$ ao Hamiltoniano inicial, além de eliminar os termos não adiabáticos ele muda o pulso de forma considerável. Como o que queremos é tirar só os termos não adiabáticos então teremos que fazer uma nova iteração. Na figura (5.5) mostra-se que ao somar o termo $H_{C D}^{(1)}(t)$ o pulso se aproxima ao pulso inicial. Neste caso $Q_{2}=70$ então o Hamiltoniano total $H^{(2)}(t)=H_{0}(t)+H_{C D}^{(1)}(t)$ já é mais adiabático do que $H_{0}(t)$.

Então, a descrição de um processo adiabático pode ser representada numa base instantânea dependente do tempo que diagonaliza o Hamiltoniano do sistema, esta base é chamada de base super-adiabática. O Hamiltoniano efetivo obtido desta forma é geralmente constituído pela soma de duas contribuições: um Hamiltoniano que é diagonal instantâneamente e outro não diagonal. O termo não diagonal contem elementos que podem impedir a evolução adiabática, mas pode ser desprezado quando $\left(Q_{n}\right)^{-1} \ll 1$. 


\subsection{Terceira Aplicação}

Vamos a considerar uma partícula com spin $1 / 2$ na presença de um campo magnético do tipo $\vec{B}(t)=B_{0} \hat{z}+B_{1}(\cos \omega t \hat{i}+\operatorname{sen} \omega t \hat{j})$ então o Hamiltoniano que representa o acoplamento spin-campo magnético é

$$
H(t)=\frac{\omega_{0}}{2} \sigma_{z}+\frac{\omega_{1}}{2}\left(\sigma_{x} \cos \omega t+\sigma_{y} \operatorname{sen} \omega t\right)
$$

Também vamos considerar que o sistema sofre uma relaxação transversal. Então a equação mestra que governa a dinâmica do sistema pode ser escrita como

$$
\dot{\rho}=i[H(t), \rho]+\alpha^{2}\left[\sigma_{z} \rho \sigma_{z}-\rho\right]
$$

ou

$$
|\dot{\rho}(t)\rangle\rangle=\mathcal{L}(t)|\rho\rangle\rangle
$$

A matriz $\mathcal{L}(t)$ fica (em uniddes de $\left.\alpha^{2}\right)$.

$$
\mathcal{L}(t)=\left(\begin{array}{cccc}
0 & 0 & 0 & 0 \\
0 & -2 & -\omega_{0}^{\prime} & \omega_{1}^{\prime} \operatorname{sen} \omega t \\
0 & \omega_{0}^{\prime} & -2 & -\omega_{1}^{\prime} \cos \omega t \\
0 & -\omega_{1}^{\prime} \operatorname{sen} \omega t & \omega_{1}^{\prime} \cos \omega t & 0
\end{array}\right)
$$

sendo $\omega_{0}^{\prime}=\omega_{0} / \alpha^{2}$ e $\omega_{1}^{\prime}=\omega_{1} / \alpha^{2}$. Os autovalores da matriz $\mathcal{L}(t)$ são

$$
\begin{aligned}
& \lambda_{1}= \\
& \lambda_{i}^{3}+4 \lambda_{i}^{2}+\left(4+\omega_{1}^{\prime 2}+\omega_{0}^{\prime 2}\right) \lambda_{i}+2 \omega_{1}^{\prime 2}=\quad 0 \quad i=2,3,4 .
\end{aligned}
$$

O correspondente autovetor direita e esquerda para $\lambda_{1}=0$ é

$$
\left.\left.\left|D_{1}\right\rangle\right\rangle=\left(\begin{array}{l}
1 \\
0 \\
0 \\
0
\end{array}\right) \quad\left|E_{1}\right\rangle\right\rangle=\left(\begin{array}{llll}
1 & 0 & 0 & 0
\end{array}\right)
$$


e para $i=2,3,4$

$$
\left.\left.\left|D_{i}\right\rangle\right\rangle=\frac{1}{\sqrt{M_{i}}}\left(\begin{array}{c}
a_{i} \\
b_{i} \\
c_{i} \\
d_{i}
\end{array}\right) \quad\left|E_{i}\right\rangle\right\rangle=\frac{1}{\sqrt{M_{i}}}\left(\begin{array}{cccc}
A_{i} & B_{i} & C_{i} & D_{i}
\end{array}\right)
$$

sendo

$$
\begin{array}{llc}
a_{i} & = & 0 \\
b_{i} & = & \omega_{0}^{\prime} \cos \omega t+\left(2+\lambda_{i}\right) \operatorname{sen} \omega t \\
c_{i} & = & \omega_{0}^{\prime} \operatorname{sen} \omega t-\left(2+\lambda_{i}\right) \cos \omega t \\
d_{i}= & -\frac{\left(2+\lambda_{i}\right) \omega_{1}^{\prime}}{\lambda_{i}} \\
A_{i}= & 0 \\
B_{i}= & \omega_{0}^{\prime} \cos \omega t-\left(2+\lambda_{i}\right) \operatorname{sen} \omega t \\
C_{i}= & \omega_{0}^{\prime} \operatorname{sen} \omega t+\left(2+\lambda_{i}\right) \cos \omega t \\
D_{i}= & -\frac{\left(2+\lambda_{i}\right) \omega_{1}^{\prime}}{\lambda_{i}}
\end{array}
$$

e

$$
M_{i}=A_{i} a_{i}+B_{i} b_{i}+C_{i} c_{i}+D_{i} d_{i}=\frac{\omega_{0}^{\prime 2} \lambda_{i}^{2}+\left(2+\lambda_{i}\right)^{2}\left(\omega_{1}^{\prime 2}-\lambda_{i}^{2}\right)}{\lambda_{i}^{2}} .
$$

Pode-se mostrar que $\left\langle\left\langle E_{j} \mid D_{i}\right\rangle\right\rangle=\delta_{i j}$. Logo a condição adiabática para nosso caso é

$$
\begin{gathered}
C_{j i}=\left|\frac{\left\langle\left\langle E_{j}|\dot{\mathcal{L}}| D_{i}\right\rangle\right\rangle}{\omega_{i j}^{2}}\right|=\left|\frac{\omega \omega_{1}^{\prime 2} \omega_{0}^{\prime}}{\sqrt{M_{j} M_{i}}\left(\lambda_{i}-\lambda_{j}\right)^{2}}\left(\frac{2+\lambda_{j}}{\lambda_{j}}-\frac{2+\lambda_{i}}{\lambda_{i}}\right)\right| \ll 1 \\
C_{j i}=\left|\frac{\omega \omega_{0}^{\prime}\left[4+\lambda_{i}+\lambda_{j}\right]}{\sqrt{M_{j} M_{i}}\left(\lambda_{i}-\lambda_{j}\right)}\right| \ll 1 \quad i, j=2,3,4 .
\end{gathered}
$$

Podemos que ver que a condição adiabática depende linearmente de $\omega$. Além disso na figura (5.7) pode-se observar que o termo $C_{j i}$ é pequeno para valores grandes de $\omega_{0}^{\prime}$. 

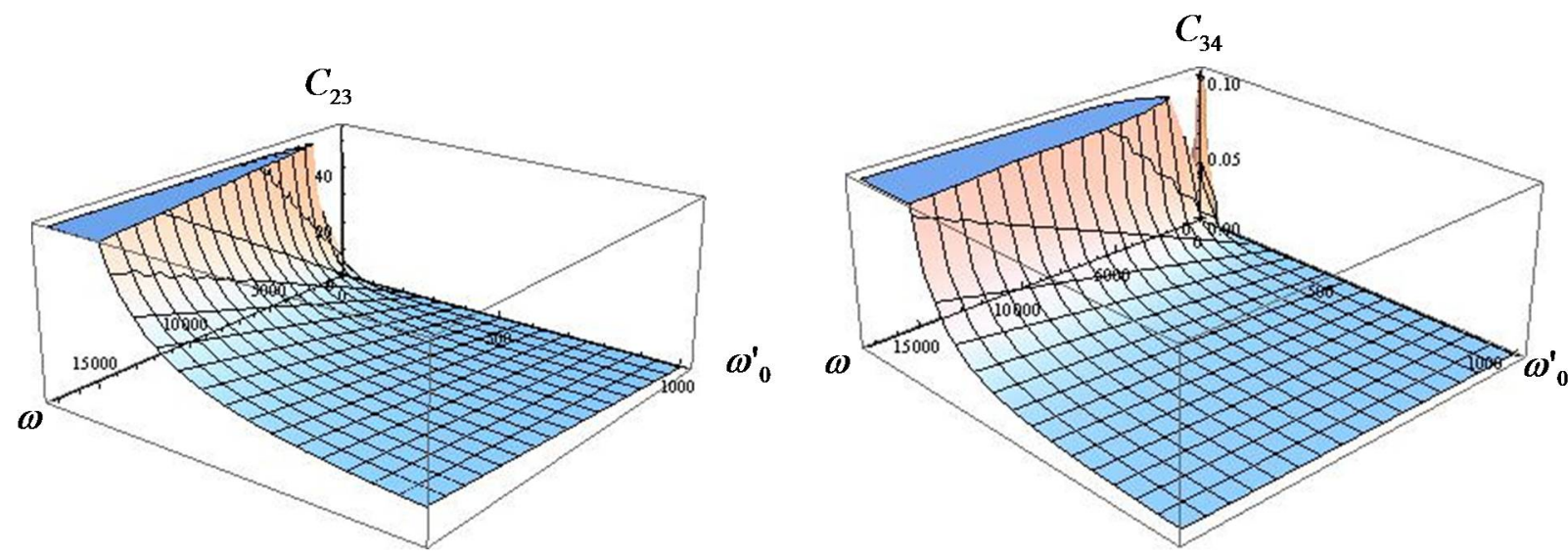

Figura 5.7-Gráfico da equação (5.3.12) em função de $\omega$ e $\omega_{0}^{\prime}=\omega_{1}^{\prime}$.

Fonte: Elaborada pelo autor.

Sendo a matriz de transformação

$$
U(t)=\left(\begin{array}{cccc}
1 & 0 & 0 & 0 \\
0 & \frac{b_{2}}{M_{2}} & \frac{b_{3}}{M_{3}} & \frac{b_{4}}{M_{4}} \\
0 & \frac{c_{2}}{M_{2}} & \frac{c_{3}}{M_{3}} & \frac{c_{4}}{M_{4}} \\
0 & \frac{d_{2}}{M_{2}} & \frac{d_{3}}{M_{3}} & \frac{d_{4}}{M_{4}}
\end{array}\right) \quad U^{-1}(t)=\left(\begin{array}{cccc}
1 & 0 & 0 & 0 \\
0 & \frac{B_{2}}{M_{2}} & \frac{C_{2}}{M_{2}} & \frac{D_{2}}{M_{2}} \\
0 & \frac{B_{3}}{M_{3}} & \frac{C_{3}}{M_{3}} & \frac{D_{3}}{M_{3}} \\
0 & \frac{B_{4}}{M_{4}} & \frac{C_{4}}{M_{4}} & \frac{D_{4}}{M_{4}}
\end{array}\right)
$$

Ao fazer a transformação da matriz $\mathcal{L}(t)$, tal que $\left.|\rho\rangle\rangle_{J}=U^{\dagger}|\rho\rangle\right\rangle$, obtemos:

$$
\left.\left.\left[\mathcal{L}_{J}(t)+\mathcal{L}_{D}(t)+\mathcal{L}_{N D}(t)\right]|\rho\rangle\right\rangle_{J}=|\dot{\rho}\rangle\right\rangle_{J}
$$

onde cada termo pode-se obter das equações (5.3.9)

$$
\begin{gathered}
\mathcal{L}_{J}(t)=\left(\begin{array}{cccc}
0 & 0 & 0 & 0 \\
0 & \lambda_{2} & 0 & 0 \\
0 & 0 & \lambda_{3} & 0 \\
0 & 0 & 0 & \lambda_{4}
\end{array}\right) \\
\mathcal{L}_{D}(t)=\left(\begin{array}{cccc}
0 & 0 & 0 & 0 \\
0 & -\frac{2 \omega_{0}^{\prime} \omega\left(2+\lambda_{2}\right)}{M_{2}} & 0 & 0 \\
0 & 0 & -\frac{2 \omega_{0}^{\prime} \omega\left(2+\lambda_{3}\right)}{M_{3}} & 0 \\
0 & 0 & 0 & -\frac{2 \omega_{0}^{\prime} \omega\left(2+\lambda_{4}\right)}{M_{4}}
\end{array}\right)
\end{gathered}
$$




$$
\mathcal{L}_{N D}(t)=\left(\begin{array}{cccc}
0 & 0 & 0 & 0 \\
0 & 0 & \frac{\omega_{0}^{\prime} \omega \lambda_{4}}{\sqrt{M_{2} M_{3}}} & \frac{\omega_{0}^{\prime} \omega \lambda_{3}}{\sqrt{M_{2} M_{4}}} \\
0 & \frac{\omega_{0}^{\prime} \omega \lambda_{4}}{\sqrt{M_{2} M_{3}}} & 0 & \frac{\omega_{0}^{\prime} \omega \lambda_{2}}{\sqrt{M_{3} M_{4}}} \\
0 & \frac{\omega_{0}^{\prime} \omega \lambda_{3}}{\sqrt{M_{2} M_{4}}} & \frac{\omega_{0}^{\prime} \omega \lambda_{2}}{\sqrt{M_{3} M_{4}}} & 0
\end{array}\right)
$$

e o termo contradibático pode ser encontrado a partir de

$$
\begin{gathered}
\mathcal{L}_{C D}(t)=-U(t) \mathcal{L}_{N D}(t) U^{\dagger}(t) . \\
\mathcal{L}_{C D}(t)=\left(\begin{array}{cccc}
0 & 0 & 0 & 0 \\
0 & h_{22} & h_{23} & h_{24} \\
0 & h_{32} & h_{33} & h_{34} \\
0 & h_{42} & h_{43} & h_{44}
\end{array}\right)
\end{gathered}
$$

sendo

$$
\begin{aligned}
& h_{22}=\frac{B_{4}}{M_{4}}\left(\frac{b_{3} \lambda_{2}}{M_{3}}+\frac{b_{2} \lambda_{3}}{M_{2}}\right)+\frac{B_{3}}{M_{3}}\left(\frac{b_{4} \lambda_{2}}{M_{4}}+\frac{b_{2} \lambda_{4}}{M_{2}}\right)+\frac{B_{2}}{M_{2}}\left(\frac{b_{4} \lambda_{3}}{M_{4}}+\frac{b_{3} \lambda_{4}}{M_{3}}\right) \\
& h_{32}=\frac{B_{4}}{M_{4}}\left(\frac{c_{3} \lambda_{2}}{M_{3}}+\frac{c_{2} \lambda_{3}}{M_{2}}\right)+\frac{B_{3}}{M_{3}}\left(\frac{c_{4} \lambda_{2}}{M_{4}}+\frac{c_{2} \lambda_{4}}{M_{2}}\right)+\frac{B_{2}}{M_{2}}\left(\frac{c_{4} \lambda_{3}}{M_{4}}+\frac{c_{3} \lambda_{4}}{M_{3}}\right) \\
& h_{42}=\frac{B_{4}}{M_{4}}\left(\frac{d_{3} \lambda_{2}}{M_{3}}+\frac{d_{2} \lambda_{3}}{M_{2}}\right)+\frac{B_{3}}{M_{3}}\left(\frac{d_{4} \lambda_{2}}{M_{4}}+\frac{d_{2} \lambda_{4}}{M_{2}}\right)+\frac{B_{2}}{M_{2}}\left(\frac{d_{4} \lambda_{3}}{M_{4}}+\frac{d_{3} \lambda_{4}}{M_{3}}\right) \\
& h_{23}=\frac{C_{4}}{M_{4}}\left(\frac{b_{3} \lambda_{2}}{M_{3}}+\frac{b_{2} \lambda_{3}}{M_{2}}\right)+\frac{C_{3}}{M_{3}}\left(\frac{b_{4} \lambda_{2}}{M_{4}}+\frac{b_{2} \lambda_{4}}{M_{2}}\right)+\frac{C_{2}}{M_{2}}\left(\frac{b_{4} \lambda_{3}}{M_{4}}+\frac{b_{3} \lambda_{4}}{M_{3}}\right) \\
& h_{33}=\frac{C_{4}}{M_{4}}\left(\frac{c_{3} \lambda_{2}}{M_{3}}+\frac{c_{2} \lambda_{3}}{M_{2}}\right)+\frac{C_{3}}{M_{3}}\left(\frac{c_{4} \lambda_{2}}{M_{4}}+\frac{c_{2} \lambda_{4}}{M_{2}}\right)+\frac{C_{2}}{M_{2}}\left(\frac{c_{4} \lambda_{3}}{M_{4}}+\frac{c_{3} \lambda_{4}}{M_{3}}\right) \\
& h_{43}=\frac{C_{4}}{M_{4}}\left(\frac{d_{3} \lambda_{2}}{M_{3}}+\frac{d_{2} \lambda_{3}}{M_{2}}\right)+\frac{C_{3}}{M_{3}}\left(\frac{d_{4} \lambda_{2}}{M_{4}}+\frac{d_{2} \lambda_{4}}{M_{2}}\right)+\frac{C_{2}}{M_{2}}\left(\frac{d_{4} \lambda_{3}}{M_{4}}+\frac{d_{3} \lambda_{4}}{M_{3}}\right) \\
& h_{24}=\frac{D_{4}}{M_{4}}\left(\frac{b_{3} \lambda_{2}}{M_{3}}+\frac{b_{2} \lambda_{3}}{M_{2}}\right)+\frac{D_{3}}{M_{3}}\left(\frac{b_{4} \lambda_{2}}{M_{4}}+\frac{b_{2} \lambda_{4}}{M_{2}}\right)+\frac{D_{2}}{M_{2}}\left(\frac{b_{4} \lambda_{3}}{M_{4}}+\frac{b_{3} \lambda_{4}}{M_{3}}\right) \\
& h_{34}=\frac{D_{4}}{M_{4}}\left(\frac{c_{3} \lambda_{2}}{M_{3}}+\frac{c_{2} \lambda_{3}}{M_{2}}\right)+\frac{D_{3}}{M_{3}}\left(\frac{c_{4} \lambda_{2}}{M_{4}}+\frac{c_{2} \lambda_{4}}{M_{2}}\right)+\frac{D_{2}}{M_{2}}\left(\frac{c_{4} \lambda_{3}}{M_{4}}+\frac{c_{3} \lambda_{4}}{M_{3}}\right) \\
& h_{44}=\frac{D_{4}}{M_{4}}\left(\frac{d_{3} \lambda_{2}}{M_{3}}+\frac{d_{2} \lambda_{3}}{M_{2}}\right)+\frac{D_{3}}{M_{3}}\left(\frac{d_{4} \lambda_{2}}{M_{4}}+\frac{d_{2} \lambda_{4}}{M_{2}}\right)+\frac{D_{2}}{M_{2}}\left(\frac{d_{4} \lambda_{3}}{M_{4}}+\frac{d_{3} \lambda_{4}}{M_{3}}\right)
\end{aligned}
$$

Se somamos este termo à equação (5.3.3) se eliminan os efeitos não adiabáticos, do mesmo jeito que se fez no sistema fechado 


\section{Conclusões e Perspectivas}

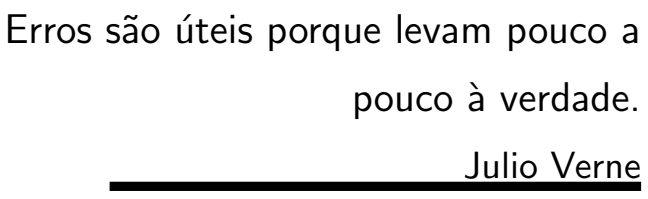

O objetivo desta dissertação foi estudar a adiabaticidade e superadiabaticidade para sistemas abertos e fechados no contexto da Ressonância Magnética Nuclear.

Agora, uma boa descrição de qualquer sistema em mecânica quântica pode ser bastante simplificada pela escolha de um quadro de referência adequado em que o correspondente $\mathrm{Ha}$ miltoniano seja diagonal. Assim, a descrição de um processo adiabático pode ser representada numa base instantânea dependente do tempo que diagonaliza o Hamiltoniano do sistema, e esta base é chamada de base adiabática $(5,33,37)$. O Hamiltoniano efetivo obtido desta forma é geralmente constituído pela soma de duas contribuições: um Hamiltoniano que é diagonal instantaneamente e o acoplamento adiabático. Este último termo não diagonal contém elementos que podem impedir a evolução adiabática. O acoplamento adiabático pode ser desprezado quando o Hamiltoniano varia muito lentamente e se isso acontece o processo é considerado adiabático. Isto nunca acontece na prática, ese efeito é muito difícil de ser observado, pois no momento da análise e concepção de processos adiabáticos as correções não adiabáticas devem ser consideradas. Em uma série de artigos $(6,30,38,39)$, Berry mostrou que o Hamiltoniano no quadro adiabático pode ser re-transformado iterativamente em outros quadros chamados superadiabáticos. Somente no quadro superadiabatico pode ser avaliada com verdadeira eficácia a aproximação adiabática, já que neste quadro se pode saber quais são os termos não adiabáticos que causam a perda de controle da dinâmica do sistema durante a evolução. Cada iteração superadiabática pode ser utilizada também para gerar um termo chamado contradiabático (40-42), que quando se adiciona ao Hamiltoniano inicial faz que a dinâmica aproximada torne-se exata. Além se podem gerar atalhos para a adiabaticidade, ou seja, se pode obter um Hamiltoniano que em um tempo finito mantenha as mesmas populações finais que a dinâmica adiabática inicial. 
Pretendemos, comprovar experimentalmente a condição da adibaticidade nos sistemas aberto e fechado no contexto de RMN, além de comprovar a otimização do processo adiabático em Ressonância Magnética seguindo trajetórias nos quadros superadiabáticos. 


\section{REFERÊNCIAS}

1 MESSIAH, A. Quantum mechanics. Amsterdam: North Holland, 1962.

2 GRIFFITHS, D. J. Introduction to quantum mechanics. New York: Prentice Hall, 1995.

3 BORN, M.; FOCK, V. Beweis des adiabatensatzes. Zeitschrift fur Physik, v. 51, n. 3-4, p. 165-180, 1916.

4 EHRENFEST, P. Adiabatische invarianten und quantentherie. Annalen der Physik, v. 356, n. 19, p. 327-352, 1916.

5 LIM, R.; BERRY, M. V. Superadiabatic tracking of quantum evolution. Journal of Physics A, v. 24, n. 14, p. $3255-3264,1991$.

6 BERRY, M. V. Quantum phase corrections from adiabatic iteration. Proceedings of the Royal Society of London A, v. 414, n. 1846, p. 31-46, 1987.

7 TANNÚS, A.; GARWOOD, M. Adiabatic pulses. Disponível em: http://eeclasses.usc.edu/ee591/library/Tannus-AdiabaticPulse.pdf $\rangle, \quad$ v. $10, \quad$ p. 423434, 1997.

8 DE GRAAF, R.; NICOLAY, K. Adiabatic rf pulses: aplications to in vivo nmr. Concepts in Magnetic Resonance, v. 9, p. 247-268, 1997.

9 ALICKI, R.; LENDI, K. Quantum dynamical semigroups and applications. Berlin: Springer, 1987. (Lecture Notes in Physics, 286).

10 BREUER, H.-P.; PETRUCCIONE, F. The theory of open quantum systems. New York: Oxford University Press, 2003.

11 SARANDY, M. S.; LIDAR, D. A. Adiabatic approximation in open quantum systems. Physical Review A, v. 71, n. 1, p. 012331-1-012331-12, 2005.

12 JONES, A. J.; VEDRAL, V.; EKERT, A.; CASTAGNOLI, G. Geometric quantum computation using nuclear magnetic resonance. Nature, London, v. 403, n. 8, p. 869-871, 2000.

13 FARHI, E.; GOLDSTONE, J. Quantum computation by adiabatic evolution. Disponível em:〈http://arXiv.org/pdf/quant-ph/0001106v1.pdf〉, Acesso em: 28 jan. 2000. 
14 COHEN-TANOUDJI, C. Quantum mechanics. New York: John Wiley \& Sons, 1977.

15 FREITAS, J. C.; BONAGAMBA, T. Os núcleos atômicos e a rmn: o modelo de camadas, o spin e os momentos electromagneticos. Rio de Janeiro: AUREMN, 1999.

16 SLICHTER, C. P. Principles of magnetic resonance. New York: Springer, 1989.

17 CALLAGHAN, P. T. Principles of nuclear magnetic resonance microscopy. New York: Oxford University Press, 1991.

18 OLIVEIRA, I. S.; BONAGAMBA, T. J.; SARTHOUR, R. S.; FREITAS, J. C. C.; DEAZEVEDO, E. R. Nmr quantum information processing. Oxford: Elsevier, 2007.

19 BONK, F. A. Tomografia de estados quânticos em sistemas de núcleos quadrupolares em spin 3/2: uma aplicação da ressonância magnética nuclear à computação quântica. 2005. p. 117. Tese (Doutorado em Física) - Instituto de Física de São Carlos, Universidade de São Paulo, São Carlos, 2005.

20 ABRAGAM, A. Principles of nuclear magnetism. New York: Oxford Science Publications, 1994.

21 LEVITT, M. H. Spin dynamics: basis of nuclear magnetic resonance. Chichester: John Wiley \& Sons, 2008.

22 NIELSEN, M. A.; CHUANG, I. L. Quantum computation and quantum information. Cambridge: Cambridge University Press, 2000.

23 DE SOUZA, A. M. Um estudo de emaranhamento e desigualdades de bell em sistemas térmicos magnéticos. 2008. p. 164. Tese (Doutorado em Física) - Centro Brasileiro de Pesquisas Físicas, Rio de Janeiro, 2008.

24 BLOCH, F. Nuclear induction. Physical Review, v. 70, n. 7-8, p. 460-474, 1946.

25 BALIAN, R. From microphysics to macrophysics: methods and applications of statistical physics. New York: Springer, 1991.

26 DE CARVALHO NETO, J. T. Tomografia de estado quântico via ressonância magnética nuclear através de rotações globais do sistema de spins. 2007. p. 170. Tese (Doutorado em Física) - Instituto de Física de São Carlos, Universidade de São Paulo, São Carlos, 2007.

27 AUCCAISE, R. Estudo de processos quânticos através da ressonância magnética nuclear em sistemas quadrupolares. 2008. p. 195. Tese (Doutorado em Física) - Centro Brasileiro de Pesquisas Físicas, Rio de Janeiro, 2008. 
28 PINTO, D. O. S. Emaranhamento térmico e simulações quânticas de sistemas magnéticos por ressonância magnética nuclear. 2009. p. 122. Tese (Doutorado em Física) - Centro Brasileiro de Pesquisas Físicas, Rio de Janeiro, 2009.

29 ERNST, R. R.; BODENHAUSEN, G.; WOKAUN, A. Principles of nuclear magnetic resonance in one and two dimensions. New York: Oxford University Press, 1997.

30 BERRY, M. V. Quantal phase factors accompanying adiabatic changes. Proceedings of the Royal Society of London A, v. 392, n. 1802, p. 45-57, 1984.

31 BROWN, J. W.; CHURCHILL, R. V. Fourier series and boundary value problems. New York: McGraw-Hill, 1993.

32 CHRUŚCINŃSK, D.; JAMIOLKOWSHI, A. Geometric phases in classical and quantum mechanics. Boston: Birkhauser, 2004.

33 TONG, D. M. Quantitative condition is necessary in guaranteeing the validity of the adiabatic approximation. Physical Review Letters, v. 104, n. 12, p. 120401-1-120401-4, 2010.

34 SARANDY, M. S.; WU, L. A.; LIDAR, D. A. Consistency of the adiabatic theorem. Quantum Information Processing, v. 3, n. 6, p. 331-339, 2004.

35 HORN, R. A.; JOHNSON, C. R. Matrix analysis. New York: Cambridge University Press, 1990.

$36 \mathrm{YI}, \mathrm{X} . \mathrm{X}$.; YU, S. X. Effective hamiltonian approach to the master equation. Journal of Optics B: Quantum and Semiclassical Optics, v. 3, n. 6, p. 372-375, 2001.

37 DESCHAMPS, M.; KERVERN, G.; MASSIOT, D.; PINTACUDA, G.; EMSLEY, L.; GRANDINETTI, P. J. Superadiabaticity in magnetic resonance. Journal of Chemical Physics, v. 129, n. 20 , p. 204110-1-204110-10, 2008.

38 BERRY, M. V. Transitionless quantum driving. Journal of Physics A: mathematical and general, v. 42, n. 36, p. 365303-1-365303-4, 2009.

39 BERRY, M. V. Histories of adiabatic quantum transitions. Proceedings of the Royal Society of London A, v. 429, n. 1876, p. 61-72, 1990.

40 IBAÑEZ, S.; CHEN, X.; ; MUGA, J. G. Shortcuts to adiabaticity by superadiabatic iterations. Disponível em: 〈http://arXiv-web.library.cornell.edu/pdf/1212.6335v1.pdf , Acesso em: 27 dez. 2012. 
41 TORRONTEGUI, E.; IBAÑEZ, S.; MARTÍNEZ-GARAOT; MODUGNO, M.; DEL CAMPO, A.; GUÉRY-ODELIN, D.; RUSCHHAUPT, A.; CHEN, X.; MUGA, J. G. Shortcuts to adiabaticity. Disponível em: 〈http://arXiv.org/pdf/1212.6343v1.pdf $\rangle$, Acesso em: 27 dez. 2012.

42 DERMIRPLAK, M.; RICE, S. A. Assisted adiabatic passage revisited. The Journal of Physical Chemistry B, v. 109, n. 14, p. 6838-6844, 2005.

43 VACANTI, V.; MONTANGERO, S.; PALMA, G. M.; PATERNOSTRO, M.; VEDRAL, V. Superadiabatic dynamics in open quantum systems. Disponível em: 〈http://arxiv.org/pdf/1307.6922v2.pdf), Acesso em: 29/07/2013.

44 DERMIRPLAK, M.; RICE, S. A. On the consistency, extremal, and global properties of counterdiabatic fields. The Journal of Chemical Physics, v. 129, n. 15, p. 154111-1-15411112, 2008.

45 YI, X. X.; TONG, D. M.; KWEK, L. C.; OH, C. H. Adiabatic approximation in open system: an alternative approach. Journal of Physics B, v. 40, n. 2, p. 281-291, 2007.

46 DU, J.; HU, L.; WANG, Y.; WU, J.; ZHAO, M.; SUTER, D. Experimental study of the validity of quantitative conditions in the quantum adiabatic theorem. Physical Review Letters, v. 101, n. 6, p. 060403-1-060403-4, 2008.

47 MANGIA, S.; LIIMATAINEN, T.; GARWOOD, M.; MICHAELI, S. Rotating frame relaxation during adiabatic pulses vs. conventional spin lock: simulations and experimental results at 4 t. Magnetic Resonance Imaging, v. 27, n. 8, p. 1074-1087, 2009.

48 BAUM, J.; TYCKO, R.; PINES, A. Broadband and adiabatic inversion of a two level system by phase modulated pulses. Physical Review A, v. 32, n. 6, p. 3435-3447, 1985. 